\title{
THEORETICAL DESCRIPTION OF THE ELECTRON-LATTICE INTERACTION IN MOLECULAR AND MAGNETIC CRYSTALS
}

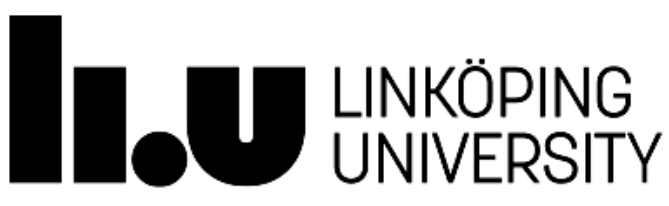

THEORETICAL AND COMPUTATIONAL PHYSICS DEPARTMENT OF PHYSICS, CHEMISTRY AND BIOLOGY LINKÖPING UNIVERSITY SE-581 83 LINKÖPING, SWEDEN 
Copyright (C) 2016 Elham Mozafari

ISBN 978-91-7685-762-5

ISSN $0345-7524$

Typeset using tufte-latex.googlecode.com

Licensed under the Apache License, Version 2.0 (the "License"); you may not use this file except in compliance with the License. You may obtain a copy of the License at http://www. apache.org/licenses/ LICENSE-2.0. Unless required by applicable law or agreed to in writing, software distributed under the License is distributed on an "as is" basis, without warranties or conditions of any kind, either express or implied. See the License for the specific language governing permissions and limitations under the License.

published by liu-tryck, linköping 2016

First printing, August 2016 
MOST PEOPLE SAY THAT IT IS THE INTELLECT WHICH MAKES A GREAT SCIENTIST. THEY ARE WRONG: IT IS CHARACTER.

ALBERT EINSTEIN 

To those who walked the walk with me. 



\section{Abstract}

Electron-lattice interactions are often considered not to play a major role in material's properties as they are assumed to be small, the second-order effects. This study, however, shows the importance of taking these effects into account in the simulations. My results demonstrate the impact of the electron-lattice interaction on the physics of the material and our understanding from it. One way to study these effects is to add them as perturbations to the unperturbed Hamiltonians in numerical simulations. The main objective of this thesis is to study electron-lattice interactions in molecular and magnetic crystals. It is devoted to developing numerical techniques considering model Hamiltonians and first-principles calculations to include the effect of lattice vibrations in the simulations of the above mentioned classes of materials.

In particular, I study the effect of adding the non-local electron-phonon coupling on top of the Holstein Hamiltonian to study the polaron stability and polaron dynamics in molecular crystals. The numerical calculations are based on the semi-empirical Holstein-Peierls model in which both intra (Holstein) and inter (Peierls) molecular electron-phonon interactions are taken into account. I study the effect of different parameters including intra and intermolecular electron-phonon coupling strengths and their vibrational frequencies, the transfer integral and the electric field on polaron stability. I found that in an ordered two dimensional molecular lattice the polaron is stable for only a limited range of parameter sets with the polaron formation energies lying in the range between 50 to $100 \mathrm{meV}$. Using the stable polaron solutions, I applied an electric field to the system and I observed that the polaron is dynamically stable and mobile for only a limited set of parameters. Adding disorder to the system will result in even a more restricted parameter set space for which the polaron is stable and moves adiabatically with a constant velocity. In order to study the effect of temperature on polaron dynamics in a one dimensional system, I include a random force in the Newtonian equations of motion. I found that there is a critical temperature above which the polaron destabilizes and becomes delocalized.

Moreover, I study the role of lattice vibrations coupled to magnetic degrees of freedom in finite temperature paramagnetic state of magnetic materials. Calculating the properties of paramagnetic materials at elevated temperatures is a cumbersome task. In this thesis, I present a new method which allows us to couple lattice vibrations and magnetic disorder above the magnetic transition temperature and treat them on the same footing. The method is based on the combination of disordered local moments model and $a b$ initio molecular dynamics (DLM-MD). I employ the method to study different physical properties of some model systems such as $\mathrm{CrN}$ and $\mathrm{NiO}$ in which the interaction between the magnetic and lattice degrees of freedom is very strong making them very good candidates for such a study.

I calculate the formation energies and study the effect of nitrogen defects on the electronic structure of paramagnetic $\mathrm{CrN}$ at high temperatures. Using this method I also study the temperature dependent elastic properties of paramagnetic $\mathrm{CrN}$. The results highlight the importance of taking into account the magnetic excitations and lattice vibrations in the studies of magnetic materials at finite temperatures.

A combination of DLM-MD with another numerical technique namely temperature dependent effective potential (TDEP) method is used to study the vibrational free energy and phase stability of $\mathrm{CrN}$. We found that the combination of magnetic and vibrational contributions to the free energy shifts down the phase boundary between the cubic paramagnetic and orthorhombic antiferromagnetic phases of $\mathrm{CrN}$ towards the experimental value. 
I used the stress-strain relation to study the temperature-dependent elastic properties of paramagnetic materials within DLM-MD with CrN as my model system. The results from a combination of DLM-MD with another newly developed method, symmetry imposed force constants (SIFC) in conjunction with TDEP is also presented as comparison to DLM-MD results.

I also apply DLM-MD method to study the electronic structure of $\mathrm{NiO}$ in its paramagnetic state at finite temperatures. I found that lattice vibrations have a prominent impact on the electronic structure of paramagnetic $\mathrm{NiO}$ at high temperatures and should be included for the proper description of the density of states.

In summary, I believe that the proposed techniques give reliable results and allow us to include the effects from electron-lattice interaction in simulations of materials. 


\section{Populärvetenskaplig sammanfattning}

Material spelar en mycket viktig och avgörande roll i vårt dagliga liv. Den myriad av material omkring oss, från byggnader till rymdfarkoster, är baserade på kunskap om hur materialen fungerar under olika omständigheter. Metaller, keramer, halvledare och polymerer är exempel på traditionella material. Emedan nanomaterial och biomaterial har utvecklats stark under senare år och är exampel på avancerade material. Hittills rådande sökmetod för designa nya material har till stor del baserats på försök och misstag. Men den tiden är förbi, i den värld vi lever i krävs snabbare och mer tillförlitliga metoder för att utforma nya material. Datorsimuleringar ger oss möjlighet att teoretiskt studera materialegenskaper snabbare och ger oss möjlighet att utveckla nya avancerade material.

De tekniker som finss tillgängliga för att simulera material gäller under förenklade förhållanden. Det finns flera viktiga faktorer som påverkar de fysikaliska och mekaniska egenskaperna hos ett material. Externa faktorer som applicering av ett elektriskt fält eller ökande temperatur kan förändra funktionen hos materialet genom påverkan samspelet mellan elektroner och gittret. Målet med denna avhandling ligger i att kunna simulera elektron-gitter interaktionerna genom att model Hamiltonians eller first-principles och studera materialet under sina verkliga driftsförhållanden. Huvudsakligen, studerar jag hur elektroner och gittret interagerar i två grupper av material, molekylära kristaller och magnetiska material.

I molekylära kristaller, kommer ett överskott elektroner polarisera gittret och genererar så kallade polaroner. För att studera dynamiken i dessa polaroner och förstå hur de beter sig när man applicerar ett ett elektriskt fält eller ökar temperaturen, krävs tillförlitliga numeriska metoder. Jag använder Holstein-Peierls modell där både inom och mellan molekylära interaktioner och specifikt elektron-phonon interaktioner beaktas. I magnetiska material, är situationen ännu mer komplicerad, speciellt vid höga temperaturer där de magnetiska momenten är oordnade. Att studera hur dessa magnetiska moment kopplas till gitterfrihetsgrader är en besvärlig uppgift. För att studera magnetiska material i deras hög temperatur paramagnetiska tillstånd, använder jag en teoretisk metod som bygger på en kombination av disordered local moments metod och ab initio molekyldynamik (DLM-MD).

Under de tre första kapitlen i denna avhandling ger jag en kort sammanfattning samt bakgrunden till teorin för de numeriska metoder jag använder. Sedan beskrivs de numeriska metoder som gör det möjligt för oss att studera dynamiska elektron gitter interaktioner i både molekylära och magnetiska kristaller, följt av en serie projekt som har genomförts med hjälp av dessa tekniker. 



\section{Preface}

This thesis is the summary of my research carried out in the Theorteical and Computational physics group at Linköping University from Jan. 2009 to Sep. 2016. The "Polaron"-related chapters of the thesis are based on my previous Licentiate thesis from Feb. 2013, A Theoretical Study of Charge Transport in Molecular Crystals, Thesis No. 1560.

My work has been focused on developing and testing numerical techniques to include electron-lattice interactions in simulations of molecular and magnetic crystals. The results from my work, appended to this thesis, are all published in peer reviewed journals with the exception of Paper VI, which is currently in the manuscript format.

Financial support for this work is mainly provided by the Swedish Research Council (VR). The theoretical calculations are carried out within the resources provided by the Swedish national infrastructure for computing (SNIC) at the Swedish National Supercomputer Center (NSC) located in Linköping. 



\section{Acknowledgment}

This thesis gives a short summary of my PhD studies in the Theorteical and Computational physics group at Linköping University. There are a number of people without whom this work would have not be possible.

Firstly and foremost, I would like to assert my deepest acknowledgments to my supervisor Igor A. Abrikosov for imparting knowledge and his enduring support since the first day I entered his group.

I would like to thank my co-supervisor Sven Stafström for providing me with the opportunity to start my research at Linköping university and his support specially in the first phase of my study.

Björn Alling, without your non-stop support and sharing knowledge, I would have been lost and none of this would have been possible, thank you so much.

Peter Steneteg, working with you is a pleasure. Jennifer Ullbrand, thank you for being there for me when I needed a strong shoulder and a holding hand. Naureen Ghafoor, I enjoyed your friendship along with all the good advice and experiences you shared with me. Jonas Saarimäki, thank you for being such a helpful friend. Ference Tasnádi, thank you for the very informative discussions and nice chats. Thank you dear Irina Yakimenko, Klara Aps Grönhagen, Diem-My Doung, Amal Hansson, Fengi Tai, Nina Shulumba, Olle Hellman, Johan Böhlin, Christian Asker, Parisa, Lida, Ali and Maryam, Mohsen and Marjan, Mohammad-Javad and Samira for being such kind and supportive friends. I am very grateful to my friends and colleagues in computational chemistry, specifically Mathieu Linares, as well as the ones in theoretical physics group for all the good time we have spent together. My wonderful friends and coffee-mates in the 9 O'clock coffee club, thank you all for the very fun morning chats. This list can go on for pages as I have had the pleasure of sharing my time here with so many great people, thank you all. I deeply appreciate all the help from the administrative team at IFM.

My wonderful friends in Iran, Tahereh, Maryam and Zahra, thank you for your warm messages and honest friendship during the past twenty-plus-years.

I am for sure indebted to my family, specially my beautiful mom and awesome dad, for all the emotional support and their prays. I could not wish for a better family.

Last but not the least, I would like to assert my gratitude to my wonderful husband, Hossein Fashandi for all the time he just sat there and listened to my constant complains, for being there for me, for supporting me and lightening my moments when every thing else felt so dark. There is this little guy in my heart, my son Sam, to whom I would give the biggest hugs and the warmest kisses in the world, as he fills my days with joy and love and as without him my world would not be this colorful.

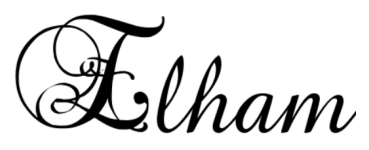

Elham Mozafari September 2016 



\section{Contents}

$1 \quad$ Introduction 19

1.1 Molecular Crystals . . . . . . . . . . . . . . . . 21

1.2 Magnetic Crystals and Paramagnetic Materials . . . . . . . . 22

1.2.1 CrN . . . . . . . . . . . . . . . . 24

$1.2 .2 \mathrm{NiO} \ldots \ldots \ldots \ldots \ldots \ldots$

2 Polaron Concept:

Theories and Methodology $\quad 27$

2.1 Classification of Polarons . . . . . . . . . . . . . . . . 27

2.2 Fröhlich Theory . . . . . . . . . . . . . . . . . 28

2.2.1 Weak Electron-Phonon Coupling . . . . . . . . . . . 29

2.2.2 Strong Electron-Phonon Coupling . . . . . . . . . . . 29

2.3 Small Polaron Theory . . . . . . . . . . . . . . . . . . 31

2.4 Holstein-Peierls Theory . . . . . . . . . . . . 32

2.4.1 Charge Carrier Dynamics . . . . . . . . . . 34

3 Paramagnetic State of Magnetic Materials:

Theories and Methodology 35

3.1 Density Functional Theory . . . . . . . . . . . . . . . 36

3.1.1 The Hohenberg-Kohn Theorem . . . . . . . . . . . . . . . . . . . . . . . . 38

3.1 .2 The Kohn-Sham Equations . . . . . . . . . . . . . . . . . . . . . . . . . . . . .

3.1 .3 The LDA+U Approach . . . . . . . . . . . . . . . . . . . . . . . . . . . . .

$3.2 \mathrm{Ab}$ initio Molecular Dynamics . . . . . . . . . . . . . . . . 41

3.2.1 Born-Oppenheimer Molecular Dynamics . . . . . . . 42

3.3 Disordered Local Moment Approach . . . . . . . . . . . . . 42

3.3.1 Supercell Implementations of the DLM Approach . . . 43

3.3.2 Disordered Local Moment Molecular Dynamics Approach 45 3.4 Temperature Dependent Effective Potential Method . . . . . 45

3.4.1 Symmetry Imposed Force Constants-TDEP Method . . 47

4 Results and Discussion

4.1 Holstein-Peierls Model Systems . . . . . . . . . . . . . . . . . 49

4.1.1 A Two Dimensional Molecular lattice . . . . . . . 50 
4.2 Geometry Optimization and Polaron Stability . . . . . . . 51

4.3 Polaron Dynamics in a Two-Dimensional System . . . . . . 53

4.3.1 Polaron Dynamics in the Presence of Disorder . . . . . 55

4.3.2 The Impact of the Temperature on Polaron Dynamics . 55

4.4 Role of $\mathrm{N}$ defects in Paramagnetic CrN . . . . . . . . . . 58

4.5 Vibrational free energy and phase stability of $\mathrm{CrN}$. . . . . 59

4.6 Temperature-Dependent Elastic Properties of CrN . . . . . . 60

4.7 The Electronic Structure of $\mathrm{NiO}$. . . . . . . . . . . . . . . 62

\section{Conclusion and Outlook 65}

A Resilient Propagation (RPROP) Algorithm $\quad 71$

B Brünger, Brooks and Karplu (BBK) Algorithm

$6 \quad$ Bibliography 75

Included Publications and Author's Contribution 83

Included Publications 83

Paper I $\quad 87$

Polaron stability in molecular crystals . . . . . . . . . . . . . 89

\section{Paper II 95}

Polaron dynamics in a two-dimentional Holstein-Peierls system

97

Paper III 105

Role of $\mathrm{N}$ defects in paramagnetic $\mathrm{CrN}$ at finite temperatures from first principles . . . . . . . . . . . . . 107

Paper IV

117

Finite-temperature elastic constants of paramagnetic materials within the diordered local moments picture from $a b$ initio molecular dynamics calculations . . . . . . . . . . . . 119 
Vibrational free energy and phase stability of paramagnetic and antiferromagnetic $\mathrm{CrN}$ from ab-initio molecular dynamics . 131

\section{Paper VI 139}

Effect of lattice dynamics on the electronic structure of paramagnetic $\mathrm{NiO}$ within Disordered Local Moment picture . . . 141 



\section{Introduction}

$\mathfrak{R}$ ATERIALS are of such importance to human kind that we even define different historical eras by the materials that could be processed and used in daily life during that period of time such as the stone age, the bronze age or the iron age. The myriad of materials around us, used for various applications from buildings to spacecrafts, are based on our knowledge of how a specific material or combination of materials function under specific circumstances. The prevailing search method to design new materials has been based on trial-and-error development for a long time. A process that takes far too long. With accelerating technological development, the demand and necessity of developing faster and more reliable methods to design materials has greatly increased.

No other invention has perhaps changed our world like computers. After decades of hard work and research on materials, we have moved from using vacuum tubes in mainframe machines to transistors which nowadays have given way to integrated circuits, microchips and microprocessors in computers. As a result, the computing power has steadily increased while it has become cheaper. This has provided researchers, with the ability to process and analyze huge amount of data. Computer simulations give us this unique opportunity to theoretically study materials properties in a much faster pace allowing us to design and study new advanced materials.

With the development of quantum mechanics and the Shrödinger equation during the last century, theoretical physicist, chemists and material scientists have developed computational techniques which give us the chance to study the physical properties of materials at the atomic level. The theory of lattice vibrations is one of the wellestablished chapters in modern solid state physics. In fact, very few of the astonishing achievements of the latter could have been made without a strong foundation of the former. A broad variety of physical properties in solids can be attributed to their lattice-dynamical behavior: thermal expansion, specific heats and heat conduction, charge mobilities and temperature dependence of physical properties related to electron-lattice interactions, to name a few.

The starting point to study the interplay between electrons and lattice vibrations is to consider the simplest model and solve the Shrödinger equation for a system of free and independent electrons. In reality, 
however, an electron in a solid interacts with its environment (nuclei and other electrons) through a potential $V(r)$. In a crystalline solid, ions are arranged in a regular periodic array and therefore the potential can also be considered as a periodic function with the same periodicity of the underlying Bravais lattice, $V(r+R)=V(r)$ for all Bravais lattice vectors $R[1]$. According to Bloch's theorem [1], the state of this electron has the form ${ }^{1}$

$$
\psi_{n, \mathrm{k}}(\mathrm{r})=e^{i \mathrm{k} \cdot \mathrm{r}} u_{n, \mathrm{k}}(\mathrm{r})
$$

where $\mathrm{k}$ is the wavevector, $n$ is an integer called the band index and $u_{n, \mathrm{k}}$ are the basis functions with the same periodicity of the underlying Bravais lattice and should satisfy the Schrödinger-type equation

$$
H_{\mathrm{k}} u_{n, \mathrm{k}}=E_{n, \mathrm{k}} u_{n, \mathrm{k}}
$$

in which $E_{n, \mathrm{k}}$ is the energy of a state with the band index $n$ and wavevector $\mathrm{k}$. The associated states for a given $n$ are called "band". In each band there is a relation between the energy of the state and the wavevector which is called the "band dispersion". The Hamiltonian $H_{\mathrm{k}}$ is the Hamiltonian of a system of free and independent electrons plus an additional perturbative term which can be solved within the "k.p" perturbation theory $[2,3] .^{2}$

$$
H_{\mathrm{k}}=H_{0}+H_{\mathrm{k}}^{\prime}=\frac{p^{2}}{2 m}+V+\frac{\hbar^{2} k^{2}}{2 m}+\frac{\hbar}{m} \mathrm{k} \cdot \mathrm{p} \text {. }
$$

The result of solving Eq. 1.2 with the Hamiltonian 1.3 is an expression for $E_{n, \mathrm{k}}$ and $u_{n, \mathrm{k}}$ in terms of energies and wavefunctions at $\mathrm{k}=0.3$ From energy dispersion relation we can also obtain a simplified expression for the effective mass [4]. It is therefore possible to study the interplay between the electron and the lattice considering the interaction between the electron and lattice vibrations as perturbation on top of the unperturbed Hamiltonian, at the level of model Hamiltonians and first principles calculations.

In this thesis I study two cases of electron-lattice interactions namely the electron-phonon interaction resulting in a polaron in molecular crystals and magnetic excitations coupled to lattice vibrations in high temperature paramagnetic materials.

One of the important facets of electron-phonon interaction is a collective excitation called the "polaron". Heuristically, the polaron concept as a consequence of the electron-phonon interaction can be explained as follows: an electron moving in an ionic crystal polarizes the atoms in its surrounding. The atoms in turn start to oscillate, affecting the motion of the electron. These atomic oscillations are described within the phonon concept. The quantum state of the combination of the electron and the polarized phonon cloud is a quasi-particle known as polaron. Strictly speaking, polaron is an upshot of dynamic electronlattice interaction. Understanding the polaron problem plays a very important role in statistical mechanics and quantum field theory, as it is a simple example of an interaction between a non-relativistic
${ }^{1}$ Neglecting the spin-orbit coupling.

${ }^{2} \mathrm{k}$ and $\mathrm{p}$ are vectors of real numbers $\left\{k_{x}, k_{y}, k_{z}\right\}$ with dimensions of inverse length and operators $\left\{-i \hbar \frac{\partial}{\partial x},-i \hbar \frac{\partial}{\partial y},-i \hbar \frac{\partial}{\partial z}\right\}$, respectively.

${ }^{3}$ In fact, if enough terms in the perturbation expansion are considered in the study, any value of $\mathrm{k}$ in the entire Brillouin zone will result in reasonably accurate analysis. 
quantum particle and a quantum field. In order to study the polarons and to understand how they behave upon applying an electric field or increasing the temperature, we need reliable numerical methods. In my work, I have used the so-called Holstein-Peierls model to study the polarons in molecular crystals, specifically in a two-dimensional system. In Ch. 2 a short summary of Polaron theories is given and the Holstein-Peierls model is explained. Numerical details and the results will be discussed later on in Ch. 4 .

Another very interesting and important problem to study is the electron-lattice interaction in magnetic materials at finite temperatures. When it comes to numerical studies of magnetic crystals, a great progress has been made in theoretical simulations of magnetically ordered ferromagnets and antiferromagnets. In paramagnetic materials with disordered magnetic moments, however, the situation is more complicated and therefore they are simulated as non-magnetic in many studies. Despite the known facts about the interplay between the magnetic moments and chemical effects for the past few decades $[5,6]$, their finite temperature behavior is not studied and the effect of the coupling between the magnetic excitations and lattice vibrations at high temperatures has often been assumed to be small. It is shown that in most cases, local magnetic moments survive above the magnetic transition temperature and thus, a proper theoretical study should include magnetic disorder, in particular, just below or above the Curie (Néel) temperature. In my simulations of paramagnetic state of magnetic materials, the magnetic disorder is considered within the disordered local moments model (DLM) $[7,8,9,10,11,12]$ and lattice vibrations at finite temperature are treated within $a b$ initio molecular dynamics (MD). The combination of these two approaches is called DLM-MD method and is described in details in Ch. 2.4.1 after a short summary of the theoretical background. The obtained results are discussed shortly in Ch. 4 .

In what follows, I give a very short introduction about the two classes of materials that I have used in my studies.

\subsection{Molecular Crystals}

Molecular crystals (MCs) and specially organic molecular crystals (OMCs) have attracted much attention during the past few decades and the research on these systems have been fueled by both academia and industry. Due to their low cost and fascinating physical characteristics, comparable energy gap to that of inorganic semiconductors, light weight and flexibility, OMCs have become good candidates to be used in technological applications such as organic light emitting diodes (OLEDs) [13, 14, 15], organic field effect transistors (OFETs) $[16,17,18]$ or organic photovoltaic cells $[19,20,21]$. The long-range order in these materials and the interplay between their $\pi$-electronic structure and geometrical structure have given rise to a developing field of research $[18,22,23,24,25]$. This specially includes investigating the physical processes and interactions in this group 
of materials that lead to their specific optical or transport properties, which is markedly different from those of conventional covalent or ionic crystals.

Although the operating principles of organic devices were initially inspired by their traditional semiconducting counterparts, the charge transport in OMCs has been a subject of strong debate ever since the pioneering experimental work of Karl et al. [26] who spent decades to synthesize and study organic molecular crystals with very high degree of purity. Despite all these outstanding experimental results, our understanding of the charge transport is still limited and requires higher level theoretical modeling and computational studies [19, 27]. The nature of charge transport in OMCs is not fully understood and the origin of the differences with inorganic semiconductors need to be explained. A very good clue for understanding and describing the charge transport in OMCs can be found in what Silinish and Čápek refer to as "mobility puzzle" [28] stating that on one hand, the mean free path of the carrier in the temperature range between $150 \mathrm{~K}$ up to room temperature is actually of the order of lattice constant $a_{0}$ $\left(a_{0} \approx l_{0}\right)$ which strongly suggests a hopping-type transport. On the other hand, the typical mobility dependence $\mu_{c}(T)$ of $\mu_{c} \propto T^{-n}$ is often supposed to speak in favor of some band-type carrier transport [28].4

Theoretical studies show that the bare electronic bandwidth of organic molecular crystals can vary and reach to $\sim 0.5 \mathrm{eV}[29,30,31]$ which is considered large compared to the thermal energy and supports the idea of band-like transport similar to the transport mechanism in inorganic semiconductors. This, however, does not suffice to indicate the transport mechanism in molecular crystals rather than resulting in high mobilities. This is because at higher temperatures, due to the vibrations, the bandwidth (i.e. the electronic coupling) is smaller than its value at zero temperature [32]. This effect is known as "band narrowing" and can be rationalized by taking the electron-phonon interactions and polaron concept $[30,33,34]$ into account. Such polaron effect may be the game-changing trigger which brings about the transport mechanism from band-like to hopping motion.

In the following chapters, the polaron concept, a short summary of polaron theories followed by computational details will be presented.

\subsection{Magnetic Crystals and Paramagnetic Materials}

Already in archaic times, people were aware of a stone found in northern Greece, Magnesia, which attracted Iron. According to Aristotle, the Greek philosopher Thales of Miletus (ca. 625-564 B.C.) even believed that this attracting materials have souls and thus are alive [35]. The first known application of magnets was in China, around year A.D. 1000, when they discovered that when a lodestone (formed by the mineral magnetite; $\mathrm{Fe}_{3} \mathrm{O}_{4}$ ) or an iron magnet is placed in a bowl of water, it would always point towards north and south resulting in the invention of the magnetic compass. However, it was not until
${ }^{4}$ The charge carrier mobility, $\mu_{c}$ is defined as the ratio between the velocity of the charge, $v$ and the electric field strength, $E$.

$$
\mu_{c}=v / E \text {. }
$$


the 12th century when they started using compass in their ships from where it spread to Europe through Arabs. Gilbert, in 1600, found that in order to produce strong magnets, it is necessary to use the right kind of Iron [36]. Gilbert's work was the first truly scientific approach towards studying magnetism and electricity. In 1820 Hans Christian Ørsted accidentally discovered that the electric current moves a compass needle. This discovery led to a spate of experiments resulting in the invention of the first electromagnet and electric motor. Later on in early 1831 , Faraday formulated the induction principle which in turn led to the introduction of electromagnetism as a new scientific discipline.

In the beginning of the 19th century, James Clerk Maxwell, provided the theoretical foundations of the electromagnetism by formulating Maxwell's equations showing that electricity and magnetism are different aspects of the same fundamental force field. Using the ideas of Boltzman's statistical thermodynamics it was then possible to develop microscopic models of the magnetic properties of atoms, molecules and later on solids.

Our current understanding of magnetism in condensed matter physics, originates from the work of Pierre Curie (1859-1906) and his wife Madame Marie Skłodowska-Curie (1867-1934). Curie carried out a series of experiments on the effect of temperature on materials such as iron and they observed that above a critical temperature, the magnetism disappears promptly. 5 To explain this spontaneous magnetic ordering, Pierre Weiss (1865-1940), proposed that there exist an internal molecular field which is proportional to the average magnetization and is responsible for spontaneous aligning of the micromagnets in the magnetic material. With the birth of quantum mechanics, more theoretical models were proposed. Great progress is made in theoretical studies and simulation of magnetic materials with ordered magnetic moments such as ferromagnets with all the spins pointing in the same direction or aniferromagnets with moments pointing antiparallel to each other in neighboring planes. The paramagnetic state of magnetic materials in which the magnetic moments are disordered has received less attention even though the effect of the interplay between the magnetic and chemical effects in magnetic materials has been known for decades [6,5]. However, these effects have been considered to be too small and not needed to be taken into account in phase stability simulations. Moreover, the paramagnetic state has been simulated as non-magnetic in many works and no differentiation has been made between the two terms which in turn could lead to erroneous conclusions [37]. In fact, in many cases local magnetic moments survive above the transition temperature. This will seriously affect the outcome of the theoretical simulations [38]. Thus the proper description of magnetic excitations is of crucial importance specially just below or above the Curie temperature [39].

Early attempts to treat magnetism in magnetic materials can be classified in two extreme limits [40]

- the localized limit for which the magnetic moments and their
${ }^{5}$ If a molecule has a magnetic moment with a magnitude $\mu_{m}$, the paramagnetic susceptibility of an ensemble (N) of such molecules is defined as

$$
\chi_{\text {para }}=N \mu_{m} / 3 k_{B} T,
$$

with $k_{B}$ being the Boltzman constant and $\mathrm{T}$ is the temperature. This is known as Curie's law and describes the susceptibility of all systems in the classical high temperature limit. 
fluctuations are localized with large and fixed amplitudes.

- the itinerant limit (band electrons) for which the magnetic moments and their fluctuations are delocalized and their amplitudes vary with temperature.

The first quantum mechanical formulation of two localized spins interacting with each other via an exchange parameter (often called the exchange integral), leading to magnetic order, was developed by Heisenberg [41]. Within the Heisenberg model both ferromagnetic and antiferromagnetic ordering could be treated.

The development of the band theory of electrons, paved the way for understanding the magnetism of itinerant electrons and is best described within the Stoner model [42, 43].

Despite all the effort, one very crucial unresolved problem was the temperature dependence of solid state magnetism. While the temperature dependence of magnetic excitations in localized moment models became clear at early stages, it took some time to find a solution for itinerant electrons models. Following the idea of including temperature in free electron gas model, Stoner applied the Fermi-Dirac distribution to incorporate the temperature effect into the itinerant electron model. However, the respective Curie temperatures turned out to be larger by a factor of 4-8 [40]. Additionally, the inverse susceptibility above the Curie temperature showed a $T^{2}$ dependence as opposed to the experimentally observed linear behavior [44]. To solve the existing controvercy between the localized and itinerant models, Moriya proposed a method based on spin density fluctuations [45]. In his method, he takes into account the nonlocal nature of spin fluctuations resulting in a unified picture of magnetism [46].

Today, after decades of constant improvement, there exist a fairly good knowledge about the mechanisms of localized and itinerant electron magnetism, yet a practicable unified model of magnetism is not at hand. Moriya's idea has been the inspiration for the development of many first-principles approaches providing methods to study paramagnetic materials. A short review of the existing methods will be given in Ch. 2.4.1.

Among all the existing paramagnetic systems, in this thesis, we restrict ourselves to $3 d$ transition metals and specifically to $\mathrm{CrN}$ and $\mathrm{NiO}$. A brief introduction about these two systems is given in the next two sections. I would like to emphasize that even though we study a limited number of systems, our approach possesses sufficient generality to be applied to other magnetic materials in their paramagnetic state [47].

\subsection{1 $\mathrm{Cr} N$}

$\mathrm{CrN}$ is a ceramic from the family of transition metal nitrides (TMNs) which has received a considerable amount of attention during the past few years. It has been valued due its hardness, corrosion resistance and many industrial applications such as hard coatings on cutting 
tools as well as wear- and corrosion resistant coatings [48, 49, 50, 51]. $\mathrm{CrN}$ also ehxibit fascinating physical properties which makes it even more interesting to study. It has been observed that at room temperature, $\mathrm{CrN}$ is a paramagnet $(\mathrm{PM})$ with a $\mathrm{B} 1 \mathrm{NaCl}$ structure. However, below the Néel temperature of $T_{N} 280-286 \mathrm{~K}[52,53,37$, it undergoes a first-order magnetostructural transition to an orthorhombic antiferromagnet (AFM) with a small distortion from the cubic $\mathrm{B}_{1}$ structure by an angle $\alpha \approx 88.3^{\circ}$, accompanied by a discontinuous volume reduction of $\sim 0.59 \%$ [53]. It is also shown that the AFM phase can be stabilized at room temperature upon applying pressure [37]. Many theoretical investigations have been carried out on $\mathrm{CrN}$ because of its electronic and magnetic properties. However, most calculations are done treating the PM cubic phase as non-magnetic. This, in turn will result in qualitatively different electronic structures with a large peak at the Fermi level as opposed to the experimental measurements that indicate the PM phase to show semiconducting behavior $[54,55,56]$. In this thesis, I introduce a recently developed method based on the disordered local moments merged with $a b$ initio molecular dynamics (DLM-MD) [57] to study vacancy formation energies, electronic structure and elastic properties of $\mathrm{CrN}$. We have also studied the vibrational free energy and phase satbility of $\mathrm{CrN}$ using a combination of DLM-MD and temperature dependent effective potential (Papers III, IV and V). All the methods are further discussed in Ch. 2.4.1.

\subsection{2 $\mathrm{NiO}$}

From the family of transition metal oxides (TMOs), NiO has long been a subject of research. It has a Néel temperature of $T_{N}=523 \mathrm{~K}$ above which $\mathrm{NiO}$ is in its paramagnetic phase with a rocksalt cubic structure $\left(\mathrm{NaCl} \mathrm{B}_{1}\right)$. Under this temperature, $\mathrm{NiO}$ possesses an antiferromagnetic (AFM) ordering with a small rhombohedral distortion from its cubic lattice structure. Early studies based on conventional band theory predicted $\mathrm{NiO}$ to be either metallic or according to density functional theory (DFT) calculations based on local spin density approximations (LSDA) $[58,59,60]$ a small band gap insulator in contrast to the experimental measurements reporting $\mathrm{NiO}$ to be an insulator with a band gap as large as $4.3 \mathrm{eV}$. Despite the numerous theoretical studies on $\mathrm{NiO}$, its electronic structure has remained enigmatic and controversial over the past decades. There are ongoing discussions about the electronic structure of $\mathrm{NiO}$ since various levels of theory, even quite advanced ones such as the GW approximation on top of the dynamical mean field theory (GW+DMFT) [61], give different results. On the other hand, temperature effects are often studied on the level of electrons and single-site magnetic disorder while the impact of lattice vibrations in the paramagnetic state is not taken into consideration. Therefore, a benchmark with well established robust DFT-DLM in combination with molecular dynamics is called for. This is the subject of the study Paper VI. 



\section{Polaron Concept:}

\section{Theories and Methodology}

$\mathfrak{W}$ HILE moving across a semiconductor or an insulator, charge carriers, electrons and holes, interact with phonons in the lattice. This interplay between the charge carrier and phonons demonstrates itself in two major effects. First, it causes electron scattering which in turn gives rise to electrical resistance and second, it changes the properties of electrons. Indeed, the electron induces a deformation in its surrounding environment in the lattice, Fig. 2.1, meaning that a cloud of virtual phonons bound to the electron and hence follows the electron through the lattice. Such a charge carrier dressed by a cloud of virtual phonons is addressed as a "Polaron". The general concept of polarons was first introduced by Landau in 1933 [62] followed by a detailed study by Pekar in $1946[63,64]$ in which he applied the polaron concept to an electron coupled to optical phonons in a polar crystal. In principle, the type of the phonons to which the charge carrier is coupled and the strength of this coupling as well as the dimensionality (D) of the space determine the properties of the polarons. In other words, a polaron is characterized by its self-energy (binding energy) $E_{0}$, its effective mass $m^{*}$ and its response to external electric and magnetic fields. In what follows I will give a short description of the classification of polarons. I will then continue the chapter by giving an introduction to theoretical models of polaron transport in molecular crystals.

\subsection{Classification of Polarons}

Depending on the number of the phonons in the cloud $(\mathrm{N})$, polarons can be classified into two categories

- strong-coupling if $\mathrm{N} \gg 1$

- weak-coupling if $\mathrm{N} \ll 1$

Comparing the size of the polaron $r_{p}$ with the lattice spacing $a$, they can be termed as

- small polarons if $r_{p} \approx a$

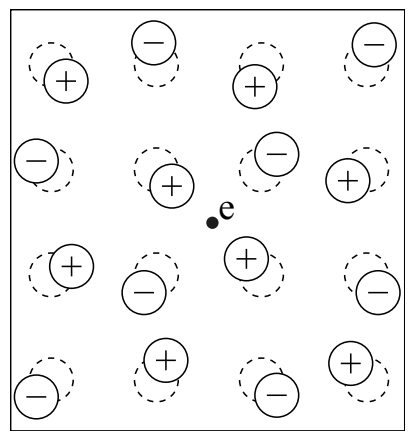

Figure 2.1: Schematic representation of a polaron formation in a polar crystal: Positive and negative ions are attracted to or repelled by the charge depending on if the charge carrier is an electron or a hole. In this figure the electron polarizes the surrounding lattice and a polaron is formed. 
- large polarons if $r_{p} \gg a$

Considering the ratio between the half-width $W$ of the electron band and the phonon frequency $\hbar \omega_{p h}$, we can sort the polarons to

- adiabatic when $W \gg \hbar \omega_{p h}$

- nonadiabatic (anti-adiabatic) when $W \ll \hbar \omega_{p h}$

It is well-known that the general polarization takes in two parts, the lattice polarization related to the displacements and the electronic polarization. In a system with closed atomic (ionic) shells, a conduction electron interacts with the electronic polarization of the crystal. When this electron moves, the electronic polarization follows it nearly without retardation, hence, it is included in the band energy of the conduction electron. To address this issue, the term "electronic polaron" $[65,66]$ is often used analogous to the polaron.

Polarons can also be put in different groups according to the electronphonon coupling mechanisms. When the local electron-phonon coupling is weak, we will have a spatially extended polaron called Fröhlisch polaron [67]. In the strong electron-phonon coupling regime, the self-induce localization caused by an excess charge is of the same order as the lattice constant. In this case the polaron is named Holstein polaron after the pioneering work of Holstein [33].

\subsection{Fröhlich Theory}

Fröhlich proposed a model Hamiltonian for the "large" polaron in which the interaction between a single electron and longitudinal optical (LO) phonons is described quantum mechanically. In Fröhlich Hamiltonian the polarization carried by LO phonons is represented by Einstein model in which the frequency is considered to be constant, $\omega_{L O} \equiv \omega_{0}$. Since it assumes only a single electron, the Hamiltonian [68] can be written as

$$
H=\frac{P^{2}}{2 m}+\hbar \omega_{0} \sum_{K} a_{K}^{\dagger} a_{K}+\sum_{K}\left(V_{k} a_{K} e^{i K \cdot r}+V_{k}^{*} a_{K}^{\dagger} e^{-i K \cdot r}\right),
$$

in which $r$ and $P$ are the conjugate coordinates of the electron. $a^{\dagger}$ and $a$ are the creation and annihilation operators, respectively, for LO phonons with wave vector $K$ and energy $\hbar \omega_{0}$. The electron-phonon interaction Fourier component $V_{k}$ is

$$
V_{k}=-i \frac{\hbar \omega_{0}}{k}\left(\frac{4 \pi \alpha}{V}\right)^{\frac{1}{2}}\left(\frac{\hbar}{2 m \omega_{0}}\right)^{\frac{1}{4}},
$$

with $\alpha$ defining the strength of the electron-phonon interaction. In a polar crystal with electronic $\varepsilon_{\infty}$ and static $\varepsilon_{0}$ dielectric constant, $\alpha$ is defined as

$$
\alpha=\frac{e^{2}}{\hbar} \sqrt{\frac{m}{2 \hbar \omega_{0}}}\left(\frac{1}{\varepsilon_{\infty}}-\frac{1}{\varepsilon_{0}}\right) .
$$


In Fröhlich theory, the unperturbed electron is assumed to move like a free-particle with an effective mass $m$. The results are independent from the particle statistics because of the single electron assumption. Moreover, the phonon modes are unaffected due to the same reason resulting in phonon self energy equal to zero [34]. The effect of the anisotropy on the effective mass as well as the degeneracy of the energy bands is not considered. These rather restricted assumptions describe the "Fröhlich polaron problem". Despite the many extensive works on solving the model Hamiltonian Eq. [69, 70, 71, 72, 73], there yet does not exist an exact solution for the problem. The best and most accurate solution available is the theory developed by Feynman [74] using a variational method based on path integrals. Some of the mathematical techniques tried out on Fröhlich polaron problem are described in the following sections.

\subsubsection{Weak Electron-Phonon Coupling}

In this regime, the electron-phonon coupling can be treated as a small perturbation in the system. The wavefunction will be slightly modified due to this interaction and the problem can be solved using the existing perturbation theories $[75,34]$. A simple and yet sufficient result is obtained from Rayleigh-Schrödinger perturbation theory [34, 68] giving the effective mass as

$$
m^{*}=\frac{m}{1-\frac{\alpha}{6}} .
$$

This means that the polaron effects make the charge carrier appear heavier than the band mass $m$. The polaron coupling constant, $\alpha$, due to lattice deformations is defined as

$$
E_{0}=-\alpha \hbar \omega_{0},
$$

with $E_{0}$ being the first order correction energy obtained from the perturbation theory. The extra mass arises from the electron-ion interaction that induces a deformation in the lattice. As the electron moves, it has to drag this deformation along with it. It takes energy to move the deformation and the drag is the actual reason for the increase in the mass with increasing the polaron coupling constant. Rayleigh-Schrödinger result predicts that something calamitous happens at $\alpha \approx 6$ : the polaron becomes localized. Eq. 2.4, therefore, implies that for the weak electron-phonon regime to be valid, $\alpha$ should be smaller than $6(\alpha<6)$. Delocalized charge carriers can thus be described within a semiclassical model with their mass being substituted by a renormalized effective mass $m^{*}$.

\subsubsection{Strong Electron-Phonon Coupling}

Historically, the strong electron-phonon coupling regime was first to be studied by Landau and Pekar in 1946. It was their study which actually preceded the word "Polaron". It is now known that their theory and its succeeding improvements are valid at large values of $\alpha$. 


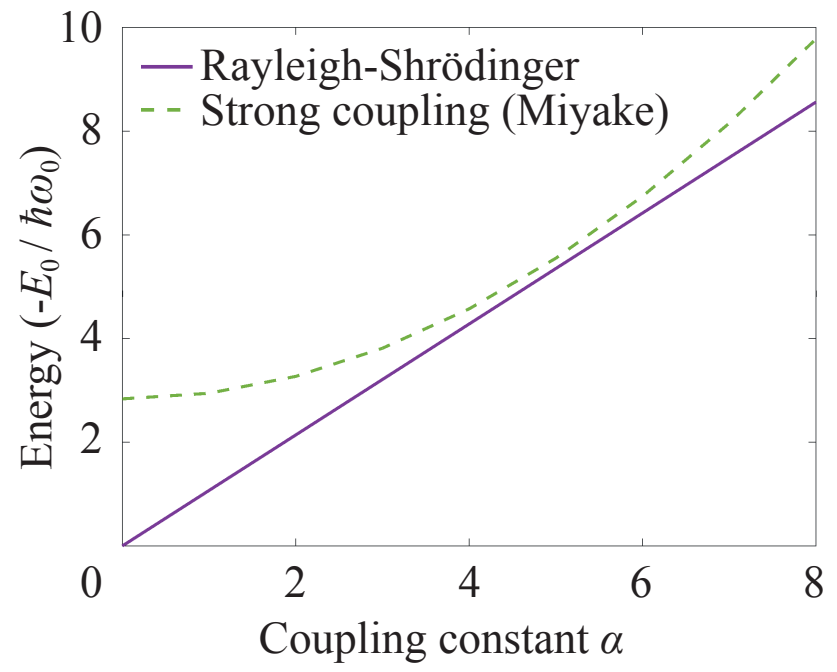

In this regime, the method of calculating the polaron constant (or the energy) is radically different from that of the weak coupling. Basically a variational calculation on a Gaussian wave function is used and the minimum energy is

$$
E_{0}=-\frac{\alpha^{2} \omega_{0}}{3 \pi}=-0.106 \alpha^{2} \omega_{0} .
$$

This equation shows that the energy is proportional to $\alpha^{2}$ rather than $\alpha$. The physics of this results can be argued in a simple way. As the electron mass is much smaller than that of the ion, it moves much faster. The motion of electron creates a charge density and in turns ions respond to this average density. A small polaron is formed as the result of a strong feedback loop. The motion of the ions creates a local potential which traps the electron in a bound state. The spatial extension of this bound state depends on the average motion of the electrons. The ions will also displace in accordance to the average motion of the electrons.

In the strong coupling regime one assumes that $\alpha$ is very large and thus the energy is expanded and evaluated as a power series in $O(1 / \alpha)$. The best available results for the strong coupling limit is derived by Mikaye [76].

$$
\lim _{\alpha \rightarrow \infty} E_{0}(\alpha)=-\omega_{0}\left[0.1085 \alpha^{2}+2.836+O\left(1 / \alpha^{2}\right)\right] .
$$

To summarize, we can say that the Rayleigh-Schrödinger expansion is valid at small values of $\alpha$ while the strong coupling covers larger values of $\alpha$. Figure 2.2 shows the ground state energy for both Rayleigh-Schrödinger and strong coupling regimes. As it is apparent the two curves almost touch each other at around $\alpha \sim 5$. This value is considered to be the crossover between two theories. Therefore,
Figure 2.2: Energy of a polaron as a function of coupling constant according to Eqs. 2.5 and 2.6. 
the correct theory to be considered for $\alpha \leq 5$ values is the RayleighSchrödinger perturbation theory whereas the strong coupling theory should be used for $\alpha>5$ values. Such a curve is precisely obtained from the "All-coupling theory" of Feynman [74].

The spatial extension of the wave function of the polaron [34] can be defined as

$$
\beta=\frac{\alpha}{3} \sqrt{\frac{4 \omega_{0} m}{\pi \hbar}} .
$$

Since $\beta$ has the dimensions of meters ${ }^{-1}$, its inverse gives an estimation for the size of the wave function. Using the electron mass and assuming $\hbar \omega_{0} \approx 0.03 \mathrm{eV}$, the polaron size will be $\beta^{-1} \sim 40 / \alpha$. Thus for $\alpha$ values between 5 and 6 , the spatial size of the wave function will be around $7 \AA$, which is about the size of an atomic unit cell, indicating a localized wave function.

Fröhlich polaron theory is based on the assumption of continuum theory for ions. Even though this theory seems reasonable for small values of coupling constant, it fails when the polaron size is of the order of atomic dimensions. Therefore, the strong coupling theory cannot be applied in real solids unless additional modifications is made to take the atomic nature of the phonons into account.

\subsection{Small Polaron Theory}

When the polaron size becomes of the same size of atomic dimensions, as occurs in the strong coupling regime, the Fröhlich polaron ${ }^{1}$ picture will not hold. This localized polaron should be considered and studied within the small polaron $[33,77]$ theory. As opposed to Fröhlich polaron model in which the motion of the charge is translationally continuous, the small polaron theory takes the priodicity of the lattice into account, assuming that the charge occupies an orbital state centered on an atomic site. The particle then moves from one site to the other. This motion can be described within the tight-binding model [1] and it can occur due to the overlap or nonorthogonality of the orbitals on adjacent sites. The prior small polaron theories are based on analytical approximations. The Hamiltonian of small polarons following the Holstein model $[33,30]$ reads as

$$
\begin{aligned}
H & =\sum_{m n} a_{m}^{\dagger} \varepsilon_{m n} a_{n}+\sum_{m k} a_{m}^{\dagger} a_{m} \hbar \omega_{K} g_{m m}^{K}\left(b_{K}^{\dagger}+b_{-K}\right) \\
& +\sum_{K} \hbar \omega_{K}\left(b_{K}^{\dagger} b_{K}+\frac{1}{2}\right),
\end{aligned}
$$

$\varepsilon_{m n}$ is the transfer integral describing the electronic coupling between orbitals at sites, $R_{m}$ and $R_{n} . a_{m}^{\dagger}\left(a_{m}\right)$ is the particle annihilation (creation) operator. $b_{K}^{\dagger}\left(b_{K}\right)$ is the annihilation (creation) operator for phonons. The Hamiltonian, Eq. 2.9, also consist of a coupling term between electrons and phonons with a coupling constant $g_{m m}^{K}$.

It is clear that both Fröhlich and Holstein Hamiltonians, Eq. 2.2 and
${ }^{1}$ Fröhlich polaron is often called large polaron. 
2.9, differ only in the form of the electron-phonon interaction. The Fröhlich Hamiltonian is written in $K$-space in which the electronphonon interaction is assumed to have a special dependence on the phonon wavevector, while the Holstein Hamiltonian is represented in real space in which the coupling constant $g$ does not have a particular form. Thus, the Fröhlich Hamiltonian may be regarded as a special form of a more general Holstein Hamiltonian in a different representation. In fact, the parameters such as the transfer integral, the phonon energy and the electron-phonon coupling determine how the polaron should be described, not the form of the Hamiltonian. If these parameters are treated correctly, one can identify different sizes of polarons and also different transport mechanisms such as band transport or hopping. From the above discussion, we see that it is more appropriate to use the Holstein Hamiltonian to describe the polarons rather than the Fröhlich Hamiltonian. However, neither the Fröhlich nor the Holstein Hamiltonians take the effect of the nonlocal electron-phonon coupling $\left(g_{m n}^{K}\right.$ with $\left.m \neq n\right)$ into account. An extension to both Fröhlich and Holstein Hamiltonians including nonlocal electron-phonon coupling (Peierls coupling) is known as the HolsteinPeierls Hamiltonian [30]. In my work, I have used this method to study the charge transport in molecular crystals numerically. In what follows, I will shortly discuss the model. The numerical details are explained later on in Ch. 4.

\subsection{Holstein-Peierls Theory}

As mentioned earlier, in Holstein model only the local electronphonon coupling is considered which acts purely on-site of the electronic excitation. To treat nonlocal electron-phonon coupling, one can use a Su-Schriffer-Heeger (SSH) [78] type model Hamiltonian. The transport theory in molecular crystals including nonlocal electronphonon coupling is investigated by many researchers, most notably by Munn et al. [79] and also Zhao et al. [80]. Later on Hannewald et al. [30] generalized the Holstein Hamiltonian by adding the nonlocal electron-phonon coupling. In their study both local (intramolecular) and nonlocal (intermolecular) electron-phonon couplings are considered and treated in a close-run. Dalla Valle and Girlando [81] also explored the possibility of separating the intra and intermolecular vibrations. They performed Raman spectroscopy on single crystals of pentacene in the temprature range between 79 to $300 \mathrm{~K}$ and compared their experimental data with the quasi harmonic lattice dynamics calculations to check the effect of the coupling between the lattice and the intramolecular vibrational modes. They found that the vibrational modes above $200 \mathrm{~cm}^{-1}$ exhibit a $100 \%$ intramolecular characteristics whereas in the intermediate range between 60 to $200 \mathrm{~cm}^{-1}$, the vibrational modes posses a significant mixing of both intra and intermolecular characteristics in an unrecognizable trend. In the former case, the nonlocal coupling can be neglected while in the latter, both local and nonlocal couplings should be considered. 
The nonlocal term cannot be treated within the band model nor within the hopping model. Using Holstein-Peierls model Hamiltonian which takes both local and nonlocal molecular interactions into account, we will be able to study the charge dynamics. In a one dimensional system consisting of $\mathrm{N}$ molecules ${ }^{2}$ the total Hamiltonian can be expressed as

$$
H=H_{e l}^{\text {intra }}+H_{e l}^{\text {inter }}+H_{\text {latt }}^{\text {intra }}+H_{\text {latt }}^{\text {inter }} .
$$

The diagonal elements of this Hamiltonian are obtained from $H_{e l}^{i n t r a}$ which is basically the Holstein model plus a disorder term.

$$
H_{e l}^{\text {intra }}=\sum_{n=1}^{N}\left(\varepsilon_{n}+A u_{n}\right) a_{n}^{\dagger} a_{n}
$$

and the off-diagonal elements of $H$ are defined within the SSH model Hamiltonian as

$$
H_{e l}^{\text {inter }}=-\sum_{n=1}^{N}\left(J_{0}+\alpha\left(v_{n+1}-v_{n}\right)\right)\left(a_{n+1}^{\dagger} a_{n}+a_{n}^{\dagger} a_{n+1}\right)
$$

where $u$ and $v$ are the intra and intermolecular displacements respectively. $\varepsilon_{n}$ is the on-site energy subjected to disorder. ${ }^{3}$ The coupling strength between a single internal phonon and the electronic system is denoted by $A$. The transfer integral $J_{0}$ value is assumed to be the same for all sites.

In semi-classical treatment that we use in this thesis, the phononic part of the Hamiltonian Eq. 2.12 is divided into two separate harmonic oscillators for describing intra and intermolecular vibrations.

$$
H_{\text {latt }}^{\text {intra }}=\frac{K_{1}}{2} \sum_{n=1}^{N} u_{n}^{2}+\frac{m}{2} \sum_{n=1}^{N} \dot{u}_{n}^{2}
$$

and

$$
H_{\text {latt }}^{\text {inter }}=\frac{K_{2}}{2} \sum_{n=1}^{N} v_{n}^{2}+\frac{M}{2} \sum_{n=1}^{N} \dot{v}_{n}^{2}
$$

where $K_{1}$ and $K_{2}$ are the force constants of the intra and intermolecular oscillators with masses $m$ and $M$, respectively.

For the charge carrier to move, a driving force has to be applied. Thus, an electric field is introduced in the system via a vector potential $\Lambda(t)=-c E t[82,83]$. The effect of the external field enters as a phase factor $\exp (i \gamma \Lambda(t))$ in the intermolecular transfer integral.

$$
J_{n+1, n}=\left(J_{0}+\alpha\left(v_{n+1}-v_{n}\right)\right) e^{i \gamma \Lambda(t)} .
$$

The parameter $\gamma \equiv e a / \hbar c$ is a constant with $e$ being the absolute value of the electric charge, $a$ the lattice constant and $c$ the speed of light.
${ }^{2}$ Note that in Holstein model every molecule represents a single site.

${ }^{3}$ For a well-ordered system, $\varepsilon_{n}=0$. 


\subsubsection{Charge Carrier Dynamics}

In the non-relativistic quantum mechanical regime, the dynamics of a charge carrier moving in an electric field is governed by time dependent Schrödinger equation (TDSE).

$$
i \hbar \frac{\partial \psi(t)}{\partial t}=\hat{H}_{e l} \psi(t) .
$$

The lattice dynamics can be defined using the Newtonian equations of motion. The force acting on a particle is equal to the negative derivative of the total energy of the system $E_{\text {tot }}=\langle\Psi|\hat{H}| \Psi\rangle$ with respect to its position.

$$
M \ddot{r}_{n}=-\nabla_{r_{n}} E_{t o t} .
$$

For a one dimensional (1D) system defined by Hamiltonian Eq.2.10 containing both electronic and phononic degrees of freedom, the equations of motions for both intramolecular and intermolecular vibrations can be expressed as

$$
m \ddot{u}_{n}=-K_{1} u_{n}-A \rho_{n, n}(t),
$$

and

$$
\begin{aligned}
M \ddot{v}_{n} & =-K_{2}\left(2 v_{n}-v_{n+1}-v_{n-1}\right) \\
& -\alpha e^{-i \gamma \Lambda(t)}\left(\rho_{n, n-1}(t)-\rho_{n+1, n}(t)\right) \\
& -\alpha e^{i \gamma \Lambda(t)}\left(\rho_{n-1, n}(t)-\rho_{n, n+1}(t)\right),
\end{aligned}
$$

respectively. $\rho$ is the density matrix.

$$
\rho_{n m}(t)=\sum_{i} \psi_{n i}(t) \psi_{i m}^{*}(t) .
$$

Having introduced the Holstein-Peierls model Hamiltonian, in Ch. 4 I will exploit more details and discuss the results of the calculations for a two dimensional system. 


\section{Paramagnetic State of Magnetic Materials: Theories and Methodology}

$\mathfrak{Z}$ ARIOUs first-principles approaches are developed in order to achieve a proper description of paramagnetic materials. As mentioned in Sec. 1.2, in my studies, I consider the general case of the $3 d$ transition metals in which the magnetism is determined through itinerant electrons which their magnetization density demonstrates a localized picture. Figure 3.1 shows the magnetization density of the orthoprhombic antifferomagnetic phase of $\mathrm{CrN}$. We see that the magnetic moments of the $\mathrm{Cr}$ atoms (large bulbs) are well localized and strongly bounded to their sites. In other words, each atom can be associated with a local magnetic moment behaving in a Heisenberg-like manner. The classical Heisenberg Hamiltonian is expressed as

$$
H_{c H}=-\sum_{i \neq j} J_{i j} \mathrm{e}_{\mathrm{i}} \mathrm{e}_{\mathrm{j}},
$$

where $J_{i j}$ is the exchange parameter. For a system of itinerant electrons, local moments become temperature dependent and are disordered above the order-disorder transition temperature which in ferromagnets is characterized by the Curie temperature $T_{C}$ and by the Néel temperature $T_{N}$ for antiferromagnets.

Moreover, local magnetic moments fluctuate in space and time due to longitudinal spin fluctuations. In a system of itinerant electrons, local magnetic moments form due to the electron-electron exchange interaction [84] and hence, the longitudinal fluctuations correspond to the itinerant nature of the electron magnetism. It is shown that these fluctuations significantly affect the high-temperature properties of magnetic materials and their thermodynamics [85]. There are several computational methods for incorporating longitudinal spin fluctuations into the simulations at the level of model Hamiltonians $[86,87,88,89,90]$ or at the level of self-consistent density functional theory (DFT) calculations in the framework of the disordered local moment picture [91].

Considering a magnetic system in its paramagnetic state, above its $T_{C}$ or $T_{N}$, the Gibbs free energy of the system will have contributions 
of freedom.

$$
G_{P M}=G_{e l}+G_{v i b}+G_{m a g} .
$$

The state-of-the-art way to deal with this problem, is to use the adiabatic approximation assuming each of the terms in Eq. 3.2 decoupled from the other two. This assumption is motivated by their very different time scales of excitations. Considering the Born-Oppenheimer approximation, one can argue that the separation between the electronic and atomic vibration degrees of freedom is well justified as the lighter electrons remain in their instantaneous ground state by adiabatically adjusting themselves to the motion of the heavier nuclei. The characteristic time for the electronic degrees of freedom, the fastest, is given by the intersite hopping and is of the order of $\sim 10^{-15} \mathrm{~s}$. The relevant time scale for the lattice vibrations can be obtained from the inverse of the Debye frequency and is of the order of $\sim 10^{-12} \mathrm{~s}$. Magnetic excitations have a time scale comparable with the inverse of the spin-wave frequency and is of the order of $\sim 10^{-13}$ s. Therefore, the time scale for the magnetic degrees of freedom is an order of magnitude faster than the vibrational ones and several orders slower than the electronic degrees of freedom.

As opposed to the low temperature regime, magnetic excitations are qualitatively different in the high-temperature paramagnetic state when other transverse and longitudinal fluctuations, such as spin flips, become dominant. The time scale of the magnetic excitations, in this case, is better estimated from the decoherence time $t_{d c}$ which is of the order of $\sim 10^{-14}-10^{-15}$ s. $^{1}$ On the other hand, in the high-temperature paramagnetic state, atomic motions should be taken into account and therefore, the proper description of the Born-Oppenheimer dynamics is obtained from the time scales of the molecular dynamics (MD) simulations and is of the order of $\sim 10^{-15}$ s. To summarize, the well-justified argument of decoupling the electronic, vibrational and magnetic contributions to the free energy for magnetically ordered systems will no longer hold or becomes questionable for the description of the paramagnetic state with disordered local magnetic moments. It is therefore important to realize that all the terms in Eq. 3.2 should be treated on the same footing in first-principles calculations of paramagnetic materials. This could be achieved via our proposed technique, the disordered local moment molecular dynamics (DLM-MD) which is going to be discussed in details later on in this chapter. I start by a short description of the basics of the density functional theory (DFT) and build my way up to introducing DLM-MD.

\subsection{Density Functional Theory}

For a many-body system consisting of many electrons and nuclei, in the absence of an external potential, the Hamiltonian can be written ${ }^{1}$ The decoherence time for the body-
centered cubic (bcc) Iron (Fe) above $T_{C}$ is
reported to be of the order $20-50 \times 10^{-15}$
$\mathrm{~s}[93]$.
Figure 3.1: Magnetization density calculated for a $2 \times 1 \times 1$ orthorhombic antiferromagnetic $\mathrm{CrN}$ unit cell. The density is demonstrated by the iso-surface at 0.43 electrons $/ \AA^{3}$. Details of the calculations are the same as the ones reported in Ref. [57]. The magnetization density of the $\mathrm{Cr}$ atoms, shown by large bulbs, is well localized. Small bulbs show the induced spin polarization at the $\mathrm{N}$ atoms. Red (blue) color displays the surplus of majority (minority) spin electrons. A more comprehensive figure of this type can be found in Ref. [92].

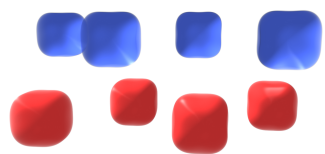

can be found in Ref. 


$$
\begin{aligned}
H & =-\frac{\hbar^{2}}{2 m_{e}} \sum_{i} \nabla_{i}^{2}-\sum_{I} \frac{\hbar^{2}}{2 M_{I}} \nabla_{I}^{2}-\sum_{i, I} \frac{Z_{I} e^{2}}{\left|r_{i}-R_{I}\right|} \\
& +\frac{1}{2} \sum_{i \neq j} \frac{e^{2}}{\left|r_{i}-r_{j}\right|}+\frac{1}{2} \sum_{I \neq J} \frac{Z_{I} Z_{J} e^{2}}{\left|R_{I}-R_{J}\right|},
\end{aligned}
$$

in which electrons are denoted by lower case subscripts and nuclei by the upper case ones. The first two terms included in the Hamiltonian 3.3 are the kinetic energy of the electrons and nuclei. The other terms are the contributions from the electron-nucleus, electron-electron and nucleus-nucleus interactions, respectively. The quantum mechanical fundamental equation through which the properties of this manybody system can be obtained is the time dependent Schrödinger equation, Eq. 2.16 with the wave function

$$
\psi\left(r_{1}, r_{2}, \ldots, r_{n} ; \sigma_{1}, \sigma_{2}, \ldots, \sigma_{n} ; R_{1}, R_{2}, \ldots, R_{n}\right)=\psi(\bar{r}, \bar{\sigma}, \bar{R}, t),
$$

with the dependence on the positions $r_{i}$ and spins $\sigma_{i}$ of the electrons and the positions of nuclei $R_{I}$. In general, when there is no explicit time dependence in the Hamiltonian 3.3, we can separate the time and spatial parts, ending up with the time-independent Schrödinger equation with stationary states $\phi(\bar{r}, \bar{\sigma}, \bar{R})$ and the total energy $E$.

$$
H \phi(\bar{r}, \bar{\sigma}, \bar{R})=E \phi(\bar{r}, \bar{\sigma}, \bar{R}) .
$$

Solving Eq. 3.3 for any condensed matter system is indeed a cumbersome task. However, simplifications can be made to make this challenging task more soluble. The first simplification is to use the Born-Oppenheimer approximation which is well justified as the difference between the electrons and nuclei masses is several orders of magnitude so that the nuclei can be considered fixed in their positions. This means we can separately solve the electronic subproblem and the effect of the nuclei can be considered as a fixed external potential. Another excellent simplification is to be able to take the lattice periodicity into account through the Bloch-theorem considering only the primitive unit cell. Therefore, the problem of a many electron system is reduced to a problem of several electrons. However, even with these simplifications, solving the Schrödinger equation, for a system of more than a few electrons, is very demanding.

The fundamental tenet of the density functional theory (DFT) starts with Thomas and Fermi $[94,95]$ who suggested to use the electron density $n(r)$ as the basic variable instead of the wave functions. This was a brilliant idea as the problem of the many electron system with the wave function being at least dependent on $3 n$ coordinates $^{2}$ is reduced to the problem of a electron density being a function of only three spatial coordinates. Their pioneering theory was not successful and failed to reproduce any qualitative description of the physical aspects of matter. The modern realization of DFT originates from the work of Hohenberg and Kohn in 1964 [96], discussed in the next section. 


\subsubsection{The Hohenberg-Kohn Theorem}

The approach that Hohenberg and Kohn suggested gives the formulation of the density functional theory which can be applied to any system of interacting particles in an external potential $V_{\text {ext }}(r)$. DFT is, in principle, based on two theorems stated and proved by them that

- for any system of interacting particles in an external potential $V_{\text {ext }}(r)$, the potential $V_{\text {ext }}(r)$ is determined uniquely, except for a constant, by the ground state particle density $n_{0}(r)$.

In simple words, this theorem implies that if one can obtain the ground state density of a system, they would know every thing about that system since through the density, the corresponding unique potential, up to a constant, is known and hence the Hamiltonian of the system. How this is done is provided within the second theorem.

- A universal functional for the energy $E[n]$ in terms of the density $n(r)$ can be defined, valid for any external potential $V_{\text {ext }}(r)$. For any particular $V_{\text {ext }}(r)$, the exact ground state energy of the system is the global minimum value of this functional, and the ground state density $n(r)$ that minimizes the functional is the exact ground state density $n_{0}(r)$.

This means that the functional $E[n]$ suffices for determining the exact ground state energy and density and therefore everything about the system.

Having these two theorems in hand, we are able to solve any electronic structure problem. The total energy functional will then have the general form

$$
\begin{aligned}
E[n] & =T[n]+E_{\text {int }}[n]+\int \mathrm{d} r V_{\text {ext }}(r) n(r)+E_{I I} \\
& =F[n]+\int \mathrm{d} r V_{\text {ext }}(r) n(r)+E_{I I},
\end{aligned}
$$

where the first two terms, on the right hand side, include all the internal energies, kinetic and potential, of the interacting electronic system. The third term is the interaction energy with the external potential from the nuclei in the system and the last term is the interaction energy of the nuclei. Despite the very nice formulation, the Hohenberg and Kohn theorem has a fundamental problem that the exact form of the universal functional $F[n]$ is not known. Fortunately, just about a year after Hohenberg and Kohn's publication, a practical scheme was developed that solved the issue.

\subsubsection{The Kohn-Sham Equations}

Kohn and Sham [97] proposed a new approach in 1965 to map the problem of an interacting many-body system onto a fictitious system of non-interacting particles with an interacting density. This means one needs to solve a system of independent particles subjected to an effective potential $V_{e f f}(r)$ instead of solving a system of interacting 
particles in a pure external potential. Thus, the system is described by solving the Schrödinegr equation for one electron wave function $\varphi_{i}$.

$$
\left(-\frac{\hbar^{2}}{2 m_{e}} \nabla^{2}+V_{e f f}(r)\right) \varphi_{i}=\epsilon_{i} \varphi_{i}
$$

with the effective potential defined as

$$
V_{e f f}(r)=V_{e x t}+\int \frac{n\left(r^{\prime}\right)}{\left|r-r^{\prime}\right|} \mathrm{d} r^{\prime}+\frac{\partial E_{x c}[n(r)]}{\partial n(r)},
$$

$E_{x c}[n]$ is called the exchange-correlation functional and is basically the only term that is approximated within the Kohn-Sham approach. For $\mathrm{N}$ single-particle states $\varphi_{i}$ with energies $\epsilon_{i}$ as in Eq. 3.7, the density is easily constructed as

$$
n(r)=\sum_{i=1}^{N}\left|\varphi_{i}(r)\right|^{2} .
$$

The kinetic energy of the system is given by

$$
\left.T_{s}[n]=-\frac{\hbar^{2}}{2 m_{e}} \sum_{i=1}^{N}<\varphi_{i}\left|\nabla^{2}\right| \varphi_{i}\right\rangle .
$$

For a system of electrons with the charge density $n(r)$ interacting with itself, the classical Coulomb interaction energy (the Hartree energy) is defined as

$$
E_{\text {Hartree }}[n]=\frac{1}{2} \int \mathrm{d} r \mathrm{~d} r^{\prime} \frac{n(r) n\left(r^{\prime}\right)}{\left|r-r^{\prime}\right|} .
$$

Following the Kohn-Sham approach, the Hohenberg-Kohn expression for the ground state energy functional, Eq. 3.6, can be written in this new form

$$
E[n]=T_{s}[n]+\int \mathrm{d} r V_{\text {ext }}(r) n(r)+E_{\text {Hartree }}[n]+E_{I I}+E_{x c}[n] .
$$

In the above equation, the energy term due to the nuclei and any other external sources $\left(V_{\text {ext }}(r)\right)$, the interaction between the nuclei $\left(E_{I I}\right)$ and the Hartree energy $\left(E_{\text {Hartree }}\right)$ represent the classical Coulomb energies. The kinetic energy of the non-interacting particles and the long-range Coulomb interaction term can be calculated explicitly. The remaining $E_{x c}[n]$ term is unknown but thanks to the genius of the Kohn-Sham approach, it can be reasonably approximated as a local functional of the density [97]. This means that we can express the exchange-correlation functional as

$$
E_{x c}^{L D A}[n]=\int \mathrm{d} r n(r) \epsilon_{x c}^{h o m}([n], r) .
$$

This is the so-called local density approximation (LDA). Shortly speaking, LDA implies that the exchange-correlation energy, at each point of the space, can be calculated as the product of the density at that point and the exchange-correlation energy per unit charge of the homogeneous electron gas with the same density. Using quantum 
Monte Carlo simulations [98], the exchange-correlation energy of the homogeneous electron gas can be given analytically [99, 100].

We note that so far the expressions are obtained for a non-spin polarized system. This is addressed by von Barth and Hedin [101] in the generalized spin-polarized Kohn-Sham approach within local density approximation. In the local spin density approximation (LSDA), we should then replace the exchange-correlations $\epsilon_{x c}^{h o m}([n], r)$ in Eq. 3.13 with $\epsilon_{x c}^{h o m}\left(n^{\uparrow}(r), n^{\downarrow}(r)\right)$ and the general Hamiltonian will be written as

$$
H_{K S}^{\alpha \beta}(r)=-\frac{1}{2} \nabla^{2}+V_{K S}^{\alpha \beta}(r) .
$$

It is clear that only the exchange-correlation part is explicitly spindependent. If the magnetic moments are considered to be collinear, ${ }^{3}$ the two density matrices become diagonal and we are therefore dealing with two separate, yet coupled, scalar densities. For the noncollinear case, the spin density matrix can always be locally diagonalized.

As an approximation, LDA (LSDA) has some general problems such as underestimating the lattice spacings. Within generalized gradient approximation (GGA) [102], this issue can be corrected to some extend. GGA is, in fact, an extension to LDA in which in addition to the local density the local gradient of the density is also considered.

$$
E_{x c}^{G G A}[n]=\int \mathrm{d} r n(r) \epsilon_{x c}^{G G A}([n, \nabla n], r) .
$$

Apart from the overbinding problem, the LDA (LSDA) has other issues which are not corrected by GGA, one of which is the underestimation of the band gaps in semiconductors. Despite being quite successful in describing different properties of solid state systems, LDA (LSDA), is also not the best functional for describing the exchange-correlation in strongly correlated systems with localized states such as transition metal and rare-earth metal compounds with partially $d$ or $f$ filled shells. It fails quite dramatically in predicting the insulating character of these materials plus giving quite poor description of their other properties such as their lattice parameters, magnetic moments, vibrational frequencies, etc. One simple but rather useful method to include strong electron correlations in the simulations is the "LDA+U" [103, 104, 105] approach exploited in the next section.

\subsubsection{The LDA+U Approach}

We start by a Hubbard-type Hamiltonian [106] which is ideally suited to describe on-site Coulomb interaction of electrons localized on atomic orbitals. The $\mathrm{LDA}+\mathrm{U}$, or the " $+\mathrm{U}$ " terminology, is one of the simplest corrective approaches formulated to improve the accuracy of LDA. ${ }^{4}$ In the LDA+U method, the Hubbard Hamiltonian is used to describe strongly correlated states (localized $\mathrm{Cr}$ and $\mathrm{Ni} 3 d$-orbitals in my calculations), while the rest of the electrons are treated within the level of LDA. Assuming N number of $d$ electrons, the total Coulomb
${ }^{3}$ When the spin axis is parallel in the whole space.
${ }^{4}$ In general, the accuracy of "DFT func-
tionals" in describing the ground state
of correlated systems. 
energy due to the $d-d$ interactions is given by the expression

$$
E=\frac{U}{2} N(N-1)
$$

Due to the additive nature of the Hubbard correction, to avoid doublecounting, it is necessary to eliminate the interaction energy already contained in the LDA functional $E^{L D A}$. The total energy of the system is therefore written as

$$
E^{L D A+U}=E^{L D A}-\frac{U}{2} N(N-1)+\frac{U}{2} \sum_{i \neq j} n_{i} n_{j} .
$$

The orbital energies are obtained from the derivative of Eq. 3.17.

$$
\epsilon_{i}=\epsilon_{i}^{L D A}+U\left(\frac{1}{2}-n_{i}\right)
$$

This shows the impact of the Hubbard correction on the band structure. The qualitative effect is to shift the occupied states $\left(n_{i}=1\right)$ downward with $U / 2$ in energy while shifting the unoccupied states $\left(n_{i}=0\right)$ upward with the same amount. It is also important to stress that the actual implementation of $\mathrm{LDA}+\mathrm{U}$ including the exchange effects, as used in this work, preserves the rotational invariance [107].

\subsection{Ab initio Molecular Dynamics}

Classical molecular dynamics and the electronic structure theory was greatly extended by $a b$ initio molecular dynamics (AIMD) techniques. In fact, AIMD has revolutionized the field of computer simulations of complex systems. ${ }^{5}$ Instead of having several parameters to fit, in AIMD we just need to provide the atomic positions and the atomic number of elements and everything else is derived from the fundamental physics laws. This makes the $a b$ initio MD to be extremely accurate as compared to classical MD. There has been a widespread of applications of AIMD in materials science and chemistry and its power has lead to an explosion of activities in terms of the number of published articles [108].

In spite of its advantages, a price has to be paid for using AIMD. It has been a prohibitively expensive method, but thanks to the developments in the theory plus the exponential growth of computer power, we are now able to solve many more interesting problems.

The very first question in any molecular dynamics calculation is the question of describing or in practice approximating the interatomic interactions and forces. In $a b$ inito molecular dynamics, the forces are computed from electronic structure calculations "on-the-fly" as the molecular dynamics trajectory is generated. This implies that we need to select a particular approximation for solving the Schrödinger equation and as argued earlier in the beginning of this chapter, we use the well-known Born-Oppenheimer approximation.

${ }^{5}$ Systems wherein the existence of many different atoms or molecules give rise to the myriad of various interatomic interactions which need to be parameterized and/or the systems in which the electronic structure, hence the bonding pattern changes qualitatively during the course of the simulation. 


\subsubsection{Born-Oppenheimer Molecular Dynamics}

One way to incorporate the electronic structure into molecular dynamics simulations is to straightforwardly solve the static electronic structure problem for each of the MD steps, considering nuclei to have fixed positions at that instant of time. We then solve the time independent Schrödinger equation for the electronic structure part and concurrently treat the nuclei within the classical molecular dynamics approach. The equations governing the Born-Oppenheimer molecular dynamics (BOMD) are expressed as

$$
M_{I} \ddot{R}_{I}(t)=-\nabla_{I} \min _{\psi_{0}}\left\{<\psi_{0}\left|H_{e}\right| \psi_{0}>\right\}
$$

and

$$
E_{0} \psi_{0}=H_{e} \psi_{0},
$$

for nuclei and electrons, respectively. We note that according to Eq. 3.19 for the nuclear equation of motion, the minimum of $H_{e}$ should be reached in each BOMD step.

\subsection{Disordered Local Moment Approach}

The disordered local moment (DLM) approach, introduced by Hubbard [10, 9, 11] and Hasegawa $[7,8]$. Within this approach, the local magnetic moments are randomly distributed on the magnetic atoms (in our case, on $\mathrm{Cr}$ or Ni sites) with spin up and spin down orientations. In 1985, Gyorffy et al. [12] applied the DLM approach combined with the coherent potential approximation (CPA) to describe the paramagnetic state of ferromagnetic materials. This approach is based on the assumption that system, taking in all the electrons, get stuck for some time ${ }^{6}$ near points in its phase space which are characterized by a finite local moment with a random direction at each site and then moves rapidly to another similar point. In other words, the ergodicity is temporarily broken ${ }^{7}$ and thus the phase space is not uniformly probed in time. Another assumption that they made was to neglect the spin-orbit coupling and therefore ignoring the noncollinearity of the local moments under the assumption of complete disorder. We here note that for a system described by the classical Heisenberg Hamiltonian, Eq. 3.1, the complete disorder picture works well if only the temperature of the system is significantly larger than the strongest exchange interaction, i.e. $T \gg J_{i j}^{\max }$. Thus, within DLM picture, we are dealing with fully disordered collinear local magnetic moments that exist above the magnetic transition temperature in the paramagnetic state. Taking these simplifications into account, we are able to describe the magnetically disordered state as a pseudo-alloy of equal number of atoms having local moments with spin-up and spin-down orientations for which the total energy and electronic structure can be calculated using CPA [12].

The DLM approach provides us with the possibility of formulating a consistent thermodynamic theory based on the electronic structure
${ }^{6}$ This time is considered to be long as compared to the hopping time $\left(10^{-15}\right.$ s) yet short enough as compared to the time scale of the magnetic excitations $\left(10^{-13} \mathrm{~s}\right)$. In this work, we denote this time as the spin-flip time $t_{s f}$.

${ }^{7}$ In fact, as supposed by Gyorffy et al., the motion of the temporarily broken ergodicity can mainly be associated with the changes in the orientational configuration of the moments [12]. 
theory inaugurating a way to describe the interplay between the configurational and magnetic degrees of freedom. Particularly, Gyorffy and Stocks [109] and later on Staunton et al. [110] derived a general formalism to include the atomic short-range order in compositionally disordered phases through the second derivative of the grand potential of the electrons for a specific configuration with respect to concentration. The method is called $S^{(2)}$ and the short-range order parameter can be calculated from its lattice Fourier transform which is directly proportional to $S^{(2)}$. These studies are based on incorporating CPA into the multiple scattering theory of Korringa and Kohn and Rostocker (KKR) [111, 112]. It is argued that even though the static DLM approximation, as explained in the previous section, neglects the dynamics of the fluctuations of charge and spin fields in time and space, it still might capture an important part of the correlations [113]. The combination of DLM and CPA as implemented by Gyorffy et al. [12] can be considered as equivalent to the "Hubbard III" approximation [114] for the original many-body problem.

As discussed above, DLM-CPA is based on multiple scattering formalism. Beside, the one-electron potential is described by the spherical approximations. This means that materials with complex underlying lattice structures, alloys in which there is a large size difference between their constituting elements or systems with point defects such as vacancies and interstitials, cannot be treated properly with DLM-CPA. Indeed, CPA is applied to describe a substantially disordered system in which the atoms are sitting on the ideal underlying crystal structure and therefore is not suitable for the treatment of lattice dynamics at finite temperatures.

\subsubsection{Supercell Implementations of the DLM Approach}

Taking a step forward in this direction, Alling et al. [115] used two different supercell implementations of the DLM approach, namely special quasi-random structure (SQS) [116] and magnetic sampling method (MSM), to simultaneously treat the magnetic and vibrational degrees of freedom at finite temperatures.

The SQS approach assumes the static picture of atoms being fixed at their ideal lattice sites and therefore the spatial disorder in the orientations of local magnetic moments gives a good approximation to obtain the energetics of the combined space and time fluctuations of magnetic moments of the paramagnetic state. From the basic assumption of fixed magnetic state, it is clear that SQS will not be applicable if the lattice vibrations are to be included in the simulations. This is because in a fixed magnetic state in time, the atoms are seen to be artificially displaced from the ideal lattice sites due to the forces between atoms having different orientations of magnetic moments and different local magnetic environments. In real paramagnets, due to the spin fluctuations in time, depending on the time scales of the magnetic excitations and atomic motions, these forces are partially averaged out and suppressed but for $\mathrm{CrN}$, as argued by Filippetti and 
Hill [117] these effects are likely to be large because of the magnetic stress.

Following Ref. [115], for a classical Heisenberg system with $N$ atoms, the average spin-spin correlation function for coordination shell $\alpha$ is defined as

$$
<\phi_{\alpha}>=\frac{1}{N} \sum_{i, j \in \alpha} \mathrm{e}_{i} \mathrm{e}_{j}
$$

The energy of the magnetic state is thus written as

$$
E_{\text {mag }}=-\sum_{\alpha} J_{\alpha} n_{\alpha}<\phi_{\alpha}>; J_{\alpha}=J_{0 j} \forall j \in \alpha,
$$

where $n_{\alpha}$ is the number of atoms in the $\alpha$-th coordination shell of the lattice. From Eq. 3.22, it is clear that for the high-temperature paramagnetic system with fully disordered magnetic moments $E_{P M}=$ 0 and the average spin-spin correlation function should follow the condition

$$
<\phi_{\alpha}>=0 ; \forall \alpha \text {. }
$$

As the exchange parameter $J$ decays with the distance, we can assume that the condition 3.23 is simplified to $\left\langle\phi_{\alpha}\right\rangle=0$ only for $\alpha$ with $J_{\alpha} \neq 0$ [92]. Note that Eq. 3.23 originally deals with an infinite system while SQS approach is used to describe a finite-sized periodic supercell. One should then be careful about the limitations of SQS [118] when implemented to describe magnetic systems.

In Ref. [92], it is pointed out that in an ideal Heisenberg model system, the magnetic interactions are constant. According to Ruban and Razumovski [119] in these kind of systems, then, the way condition 3.23 is fulfilled does not matter. Therefore, we can average over a proper set of magnetic samples. Each member of this set can have an arbitrary magnetic configuration but their average should fulfill Eq. 3.23. This is the idea behind the MSM approach suggested by Alling et al. [115]. The method is called DLM-MSM in which a set of randomly generated magnetic configurations is prepared and the energetics of each is calculated separately. The running average of all these calculations is then considered as the potential energy of the paramagnetic state. In principle, in MSM the adiabatic approximation ${ }^{8}$ can be obtained if sufficient number of different magnetic samples are calculated and the forces on each atom are averaged out. In the case of $\mathrm{CrN}$, Alling et al. [115] showed that the convergence is already reached at 40 different magnetic configurations. They also showed that different approaches, the DLM-CPA, the magnetic SQS and the DLM-MSM result in almost identical potential energies.

Starting with the idea of DLM-MSM, we can implement the DLM picture in molecular dynamics framework. This is discussed in the next section. ${ }^{8}$ when the magnetic excitations are con-
sidered to be instantaneous on the time
scales of the atomic motions. 


\subsubsection{Disordered Local Moment Molecular Dynamics Approach}

Having build up the basic assumptions and considerations in the previous sections, in this section, I explain a method for molecular dynamics simulations of paramagnetic materials at finite temperatures based on ab initio molecular dynamics (AIMD) in combination with the DLM picture: the disordered local moment molecular dynamics (DLM-MD) [57]. A schematic representation of the DLM-MD algorithm is demonstrated in Fig. 3.2.

The simulation starts with the DLM idea where the magnetic atoms in the system have spatially disordered local moments. A spin-polarized molecular dynamics calculation is then run for this system with this specific spin arrangement for a time span which we denote by spin flip time $\Delta t_{s f}$. The spins are afterwards rearranged in a random manner and the MD is run for this new magnetic configuration for another $\Delta t_{s f}$. In other words, DLM-MD realizes the concept of the temporarily broken ergodicity discussed in Sec. 3.3 as it get stuck for $\Delta t_{s f}$ time at a point in phase space corresponding to a specific spin configuration and then rapidly moves to another point with another spin arrangement.

We note that the local magnetic moments are considered to be collinear and therefore can only have up and down directions but their magnitudes can vary as dictated by the self-consistent solution of the electronic structure problem at each MD step.

The number of the MD steps that is run between two consecutive spin flip times can be obtained by $N_{s f}^{M D}=\Delta t_{s f} / \Delta t_{M D}$ where $\Delta t_{M D}$ is the time step of the MD simulations, with the condition that $\Delta t_{M D}<\Delta t_{s f}$. In simple words, during DLM-MD simulations when $N_{s f}^{M D}$ steps of $\mathrm{MD}$ is executed the magnetic configuration of the system is randomized, keeping the positions and velocities of the atoms in the lattice unchanged, and the calculations continue.

It is also important to underline that as long as the condition for the applicability of the DLM ( $T \gg J_{i j}^{\max }$ in Eq. 3.1) is fulfilled, the energetics can be calculated by an arithmetic average of over different magnetic configurations. Therefore, the net magnetization should be checked to make sure that the system remains in the DLM state during the DLM-MD simulations.

\subsection{Temperature Dependent Effective Potential Method}

Within Born-von Karman theory, the potential energy surface at zero temperature is described as a Taylor expansion of the atomic displacements from their ideal positions.

$$
U=U_{0}+U_{1}+U_{2}+U_{3}+\ldots .
$$

The term $U_{1}$ vanishes due to the symmetry of the atomic sites and in quasi-harmonic approximation the anharmonic terms $U_{3}+U_{4}+\ldots$ are neglected. On the other hand, at finite temperatures, the potential energy surface can differ significantly from that of the zero

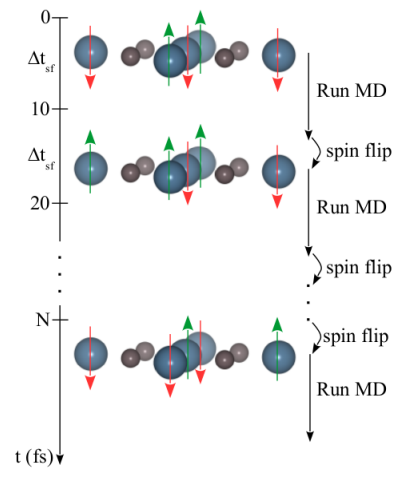

Figure 3.2: Schematic demonstration of the DLM-MD algorithm with a spin flip time $\Delta t_{s f}=10 \mathrm{fs}$. The figure shows only one layer of the atoms in the cell. Magnetic atoms are shown with big spheres and the arrows on top of them show the spin directions. 
temperature. The temperature dependent effective potential (TDEP) method $[120,121,122]$ provides us with the opportunity to obtain the finite temperature thermodynamic properties by mapping the best possible potential of the "harmonic form", including anharmonicity implicitly, at a given temperature to the true anharmonic potential given by DLM-MD calculations.

We start with a temperature-dependent model Hamiltonian to describe the lattice dynamics.

$$
H=\sum_{i \alpha} \frac{\left(p_{i}^{\alpha}\right)^{2}}{2 m_{i}}+\underbrace{U_{0}+\frac{1}{2} \sum_{i j \alpha \beta} \Phi_{i j}^{\alpha \beta} u_{i}^{\alpha} u_{j}^{\beta}}_{U^{T D E P}},
$$

where $p$ and $u$ are the momentum and the displacement. The temperature-dependent ground state energy $U_{0}$ and the second-order interatomic force constant matrix $\Phi$ are undetermined and need to be calculated. Within this model, the forces for a system of $N_{a}$ atoms are defined as

$$
\underbrace{\left(\begin{array}{c}
f_{1} \\
f_{2} \\
\vdots \\
f_{N_{a}}
\end{array}\right)}_{F_{t}^{H}}=\underbrace{\left(\begin{array}{cccc}
\overline{\bar{\Phi}}_{11} & \overline{\bar{\Phi}}_{12} & \cdots & \overline{\bar{\Phi}}_{1 N_{a}} \\
\bar{\Phi}_{21} & \overline{\bar{\Phi}}_{22} & \cdots & \overline{\bar{\Phi}}_{2 N_{a}} \\
\vdots & \vdots & \ddots & \vdots \\
\overline{\bar{\Phi}}_{N_{a} 1} & \overline{\bar{\Phi}}_{N_{a} 2} & \cdots & \overline{\bar{\Phi}}_{N_{a} N_{a}}
\end{array}\right)}_{\overline{\bar{\Phi}}} \underbrace{\left(\begin{array}{c}
u_{1} \\
u_{2} \\
\vdots \\
u_{N_{a}}
\end{array}\right)}_{U_{t}} .
$$

TDEP is based on the idea of obtaining the force constant matrices through minimizing the difference between the forces from the model Hamiltonian $F_{t}^{H}$ and the forces from DLM-MD calculations $\left\{F_{t}^{D L M-M D}\right\}$.

$$
\begin{aligned}
\min _{\bar{\Phi}} \Delta F & =\frac{1}{N_{t}} \sum_{t=1}^{N_{t}}\left|F^{D L M-M D}(t)-F^{H}(t)\right|^{2} \\
& =\frac{1}{N_{t}} \|\left(F^{D L M-M D}(1) \cdots F^{D L M-M D}\left(N_{t}\right)\right) \\
& -\bar{\Phi}\left(u^{D L M-M D}(1) \cdots u^{D L M-M D}\left(N_{t}\right)\right) \|,
\end{aligned}
$$

where $\left\{u_{t}^{D L M-M D}\right\}$ are also provided by DLM-MD calculations as well as a set of potential energies $\left\{U_{t}^{D L M-M D}\right\}$. With all these in hand, we can obtain the force constants.

In the TDEP formalism, the potential energy from the DLM-MD calculations should be equal to the potential energy from the model system $<U^{D L M-M D}>=<U^{T D E P}>$ in order to include the full anharmonicity from the DLM-MD calculations. Therefore, we consider that for every temperature $T$, the average potential energy from DLM-MD is equal to the average potential energy from TDEP. This implies that (from Eq. 3.25)

$$
U_{0}=\left\langle U^{D L M-M D}(t)-\frac{1}{2} \sum_{i j \alpha \beta} \Phi_{i j}^{\alpha \beta} u_{i}^{\alpha}(t) u_{j}^{\beta}(t)\right\rangle .
$$


Having determined the renormalized ground-state energy $U_{0}$ with implicitly including anharmonic terms and the temperature/volumedependent interatomic force constants $\Phi$, we are able to determine the full vibrational free energy including vibrational entropy in Eq. 3.2. Within TDEP formalism, the Helmholtz free energy ${ }^{9}$ for a magnetic system in a canonical (NVT) ensemble is given by

$$
F^{D L M-M D-T D E P}=U_{0}+F_{v i b}-T S_{m a g},
$$

where $S_{m a g}$ is the magnetic entropy and $F_{v i b}$ is the phonon contribution defined as

$$
F_{v i b}=\int_{0}^{\infty} g(\omega)\left\{k_{B} T\left[1-\exp \left(-\frac{\hbar \omega}{k_{B} T}\right)\right]+\frac{\hbar \omega}{k_{B} T}\right\} \mathrm{d} \omega,
$$

where $g(\omega)$ is the phonon density of states.

In a Heisenberg-like system in the paramagnetic state with collinear spin configurations, the magnetic entropy can be defined as the orientational disorder of the local magnetic moments. At temperatures considerably higher than the (isostructural) Néel temperature which is the case for $\mathrm{PM} \mathrm{CrN}$ at room temperature, the magnetic entropy of a system with local magnetic moments can be approximated by mean-field term [85]:

$$
S_{\text {mag }}=k_{B} \ln (M+1),
$$

where $M$ is the average magnitude of the local moments in units of $\mu_{B}$ and $k_{B}$ is the Boltzmann constant. In order to obtain the Gibbs free energy, what is left to be calculated is the pressure.

$$
P=-\frac{\partial F^{D L M-M D-T D E P}}{\partial V} .
$$

We are now all set to calculate the free energy. It is worth to mention TDEP takes the full symmetry of the underlying crystal structure into account reducing the computational cost. TDEP, in its original format, works for ordered materials but it can be generalized to treat substitutionally disordered alloys [123].

\subsubsection{Symmetry Imposed Force Constants-TDEP Method}

The symmetry imposed force constants temperature dependent effective potential (SIFC-TDEP) [124] can be viewed as an extension of TDEP to calculate the vibrational contribution to the free energy, Eq. 3.2, for randomly disordered alloys. Using the SQS approach [116], a substitutionally disordered alloy configuration is generated. The atoms in this system interact via the effective force constants which are obtained from TDEP as explained in Sec. 3.4. Even though in disordered alloys the symmetry of the crystal is broken but it can be imposed by treating the force constants between the alloy components of the alloy as symmetry equivalent. Thereafter, the calculation of the effective force constants $\Phi$ will not be different from that of the
${ }^{9}$ We note that the Gibbs free energy in Eq. 3.2 can be expressed as

$$
G=U-T S+P V=F+P V,
$$

with $P$ as pressure and $V$ as volume. $U$ is the internal energy, $S$ and $T$ are the entropy and the temperature, respectively. 
48 theoretical description of the electron-lattice interaction in molecular and magnetic crystals

ordered alloy done within TDEP, except that the interatomic force constants (IFCs) will be temperature/volume/concentration-dependent. In this case, the ground-state energy $U_{0}$ will also include the thermal effects of substitutional disorder in addition to the anharmonic terms. Following the same procedure as in TDEP after calculating the effective force constants, we need to obtain the phonon density of states to determine the free energy. 


\section{4}

\section{Results and Discussion}

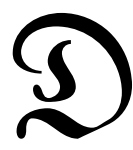

ESPITE the fact that Holstein model only considers the local electron-phonon coupling, it most likely is the best numerical model to describe polaron motion in molecular crystals up to this date. It is shown that including nonlocal electron-phonon coupling and lattice contribution will increase the hopping-type behavior $[79,80]$. The Hamiltonian, considering both local and nonlocal electron-phonon couplings concurrently is called the Holstein-Peierls Hamiltonian described earlier in Ch. 2. In this chapter I will give a more detailed description of the numerical calculations and the models I have used during the course of the first part of my research. The effect of some important factors such as disorder and temperature on polaron dynamics is also explained. All the coding and numerical calculations are done using MATLAB.

In addition, this chapter includes the description of the projects and details of the numerical calculations using the methods introduced in Ch. 2.4.I to study the paramagnetic state of magnetic materials. In my work, I have studied different properties such as the the nitrogen $(\mathrm{N})$ defect formation energies and their effect on the electronic structure of paramagnetic (PM) $\mathrm{CrN}$. The phase stability of both antiferromagnetic (AFM) and paramagnetic phases of $\mathrm{CrN}$ is also studied using a combination of DLM-MD and TDEP methods. Another project deals with the calculations of the elastic properties of paramagnetic materials at finite temperatures with $\mathrm{CrN}$ as the sample system. This chapter ends with a description of obtaining the "controversial" density of states of NiO using DLM-MD. All of the DLM-MD calculations are carried out using the Vienna ab initio simulations package (VASP) [125, 126, 127, 128].

\subsection{Holstein-Peierls Model Systems}

A one dimensional molecular chain is shown schematically in Fig. 4.1 in which both intramolecular displacements, $u_{i}$, as well as the intermolecular ones, $v_{i}$, are shown. To be more didactic, I have shown the molecules with large circles in where their constituent atoms (small circles) are embedded. The top chain display the molecular chain at the equilibrium position. Upon arrival of the charge carrier at a certain site $i$, the lattice is deformed resulting in vibrations of sites. 


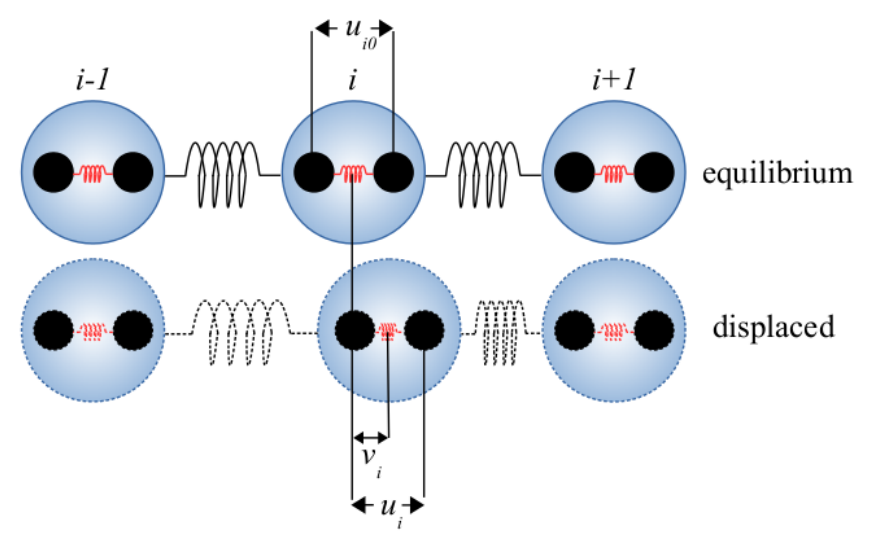

The sites will then adjust to the new position, the bottom molecular chain shown with dashed lines and shifted a bit downwards for more clarity. When the charge carrier passes, the molecule goes back to its original equilibrium position.

\subsubsection{A Two Dimensional Molecular lattice}

To get closer to real systems, one should consider higher dimensions than $1 D$. Generally speaking, molecular crystals are highly anisotropic in the sense that the in-plane electronic overlap in molecular crystals is strong as compared to the weak overlap between the planes. Therefore, choosing a two dimensional system, Fig. 4.2, in our study is not far from reality. Each molecular site is represented by two indices $(i, j)$ for $x$ and $y$ directions. The intermolecular displacements will have two components, $v_{i j}^{x}$ and $v_{i j}^{y}$. As the charge arrives at a site, both intra and intermolecular distances vibrate back and forth till the charge moves to the next site. How the charge moves and what happens in the lattice is discussed in Sec. 4.3.

The electronic Hamiltonian including both $x$ and $y$ components is expressed as

$$
\begin{aligned}
H_{e l} & =\sum_{i, j}\left(\varepsilon_{i j}+A u_{i j}\right) \hat{a}_{i j}^{\dagger} \hat{a}_{i j} \\
& +\sum_{i, j}\left(J_{i+1, j ; i j}^{x} \hat{a}_{i+1, j}^{\dagger} \hat{a}_{i j}+h . c\right) \\
& +\sum_{i, j}\left(J_{i, j+1 ; i j}^{y} \hat{a}_{i, j+1}^{\dagger} \hat{a}_{i j}+h . c\right),
\end{aligned}
$$

where

$$
J_{i+1, j ; i j}^{x}=J_{0}^{x}-\alpha\left(v_{i+1, j}^{x}-v_{i j}^{x}\right) e^{i \gamma \Lambda_{x}(t)},
$$

Figure 4.1: Schematic representation of a one dimensional molecular chain. The top chain is in the equilbrium position. The chain in the bottom is the same molecular system after the displacement, shifted downwards for clarity. The molecules are demonstrated by large circles embedding their constituent atoms, small circles. The intramolecular $u_{i}$ and intermolecular $v_{i}$ displacements are also shown.

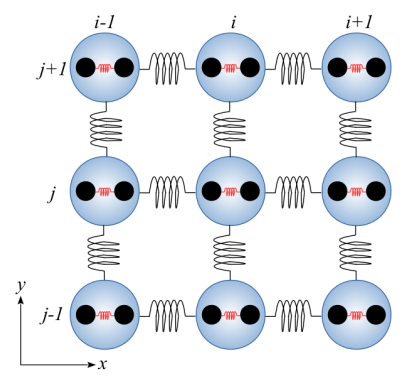

Figure 4.2: Schematic representation of a two dimensional molecular lattice. See Fig.4.1 for details. 
and

$$
J_{i, j+1 ; i j}^{y}=J_{0}^{y}-\alpha\left(v_{i, j+1}^{x}-v_{i j}^{x}\right) e^{i \gamma \Lambda_{y}(t)} .
$$

The extended lattice part of the Hamiltonian in accordance with Eqs. 2.13 and 2.14 is

$$
\begin{aligned}
H_{\text {latt }} & =\frac{K_{1}}{2} \sum_{i, j} u_{i j}^{2}+\frac{m}{2} \sum_{i j} \dot{u}_{i j}^{2} \\
& +\frac{K_{2}}{2} \sum_{i, j}\left(v_{i+1, j}^{x}-v_{i j}^{x}\right)^{2} \\
& +\frac{K_{2}}{2} \sum_{i, j}\left(v_{i, j+1}^{y}-v_{i j}^{y}\right)^{2} \\
& +\frac{M}{2} \sum_{i j}\left(\dot{v}_{i j}^{x}\right)^{2}+\left(\dot{v}_{i j}^{y}\right)^{2} .
\end{aligned}
$$

Having written the Hamiltonian, we can now solve the Newtonian equations of motion for the intramolecular displacements

$$
m \ddot{u}_{i j}=-K_{1} u_{i j}(t)-A \rho_{i j ; i j}(t),
$$

and the intermolecular displacements in both $x$ and $y$ directions

$$
\begin{aligned}
M \ddot{v}_{i j}^{x}(t) & =-K_{2}\left(2 v_{i j}^{x}(t)-v_{i+1, j}^{x}(t)-v_{i-1, j}^{x}(t)\right) \\
& -\alpha e^{-i \gamma \Lambda_{x}(t)}\left(\rho_{i j ; i-1, j}(t)-\rho_{i+1, j ; i j}(t)\right) \\
& -\alpha e^{i \gamma \Lambda_{x}(t)}\left(\rho_{i-1, j ; i j}(t)-\rho_{i j ; i+1, j}(t)\right), \\
M \ddot{v}_{i j}^{y}(t) & =-K_{2}\left(2 v_{i j}^{y}(t)-v_{i, j+1}^{y}(t)-v_{i, j-1}^{y}(t)\right) \\
& -\alpha e^{-i \gamma \Lambda_{y}(t)}\left(\rho_{i j ; i, j-1}(t)-\rho_{i, j+1 ; i j}(t)\right) \\
& -\alpha e^{i \gamma \Lambda_{y}(t)}\left(\rho_{i, j-1 ; i j}(t)-\rho_{i j ; i, j+1}(t)\right),
\end{aligned}
$$

along with TDSE, Eq. 2.16 simultaneously to obtain the dynamical behavior of the polaron as it is done in Paper II and discussed shortly in Sec. 4.3 .

\subsection{Geometry Optimization and Polaron Stability}

We start our calculations by optimizing our initial geometry using resilient propagation algorithm (RPROP) developed by Riedmiller and Braun [129]. The details of RPROP algorithm can be found in Appendix A. In order to determine the existence of the polaron, the ground state configurations for different parameter sets are determined by minimizing the total energy with respect to the intra and intermolecular displacements. The total energy in general can be defined as the summation of kinetic, potential and electronic energies of 
the system. For the ground state, the kinetic energy is zero. Therefore, in Holstein-Peierls model, the total energy can be written as

$$
E_{\text {tot }}=E_{\text {pot }}^{\text {intra+inter }}+E_{\text {elec }} .
$$

Obtaining the potential energy is straightforward.

$$
\begin{aligned}
E_{p o t} & =\frac{1}{2} K_{1} \sum_{i, j} u_{i j}^{2}+\frac{1}{2} K_{2} \sum_{i, j}\left(v_{i+1, j}^{x}-v_{i, j}^{x}\right)^{2} \\
& +\frac{1}{2} K_{2} \sum_{i, j}\left(v_{i, j+1}^{y}-v_{i, j}^{y}\right)^{2}
\end{aligned}
$$

The electronic energy is also obtained by solving the time independent Schrödinger equation (TDSE) for the ground state using the Hamiltonian 4.1.

$$
\hat{H}_{e l}\left|\psi_{0}>=E_{\text {elec }}\right| \psi_{0}>
$$

The wavefunction, $\psi$ is

$$
\psi=\sum_{i, j} C_{i, j} \varphi_{i, j}
$$

with $\varphi$ designating a set of eigenfunctions each of which satisfies the Schrödinger equation. The electronic energy will then simply be the sum over the energies of all eigenfunctions.

$$
E_{\text {elec }}=\sum_{i, j, i^{\prime}, j^{\prime}} C_{i, j} H_{i, j, i^{\prime}, j^{\prime}} C_{i^{\prime}, j^{\prime}} .
$$

Now the total energy, $E_{t o t}$, can be differentiated with respect to the intra and intermolecular positions, $R_{i, j}{ }^{1}$

$$
\frac{\partial E_{t o t}}{\partial R_{i, j}}=<\psi_{0}|\hat{H}| \psi_{0}>.
$$

The final answer is the sum of all intra and intermolecular gradients. Using RPROP algorithm this equation is solved self-consistently and the ground state geometry of the system is obtained.

Figure 4.3 shows the ground state molecular charge density of a stable 2D polaron obtained from RPROP using Holstein-Peierls Hamiltonian.

The stability of the polaron can be assessed by what is known as "polaron formation energy", $E_{p}$, and is defined as

$$
E_{p}=-\left(E_{p}^{\text {charged }}+2\left(J_{0}^{x}+J_{0}^{y}\right)\right),
$$

which is, in fact, the total energy difference between the neutral system with its molecules in their equilibrium geometries and the charged system ${ }^{2}$ with its molecules in their newly adapted equilibrium geometries. The ground state energy is equal to $\Delta J$ with $\Delta$ being the dimensions of the system [130]. In our case $\Delta=2$ and the transfer integral $J$ has two components $x$ and $y$. For the polaron to be stable, $E_{p}$ has to have a negative value $\left(E_{p}<0\right)$ for positive

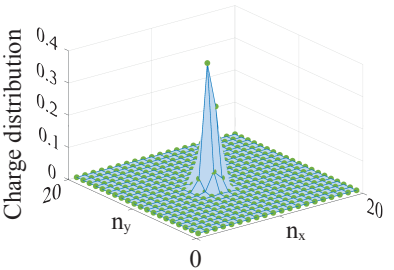

Figure 4.3: Ground state molecular charge density of a stable polaron in a two dimensional system in the HolsteinPeierls model with $A=1.5 \mathrm{eV} / \AA, \alpha=$ $0.5 \mathrm{eV} / \AA, K_{1}=10 \mathrm{eV} / \AA^{2}$ and $K_{2}=1.5$ $\mathrm{eV} / \AA^{2}$. The transfer integral value is $J_{0}^{x}=J_{0}^{y}=0.05 \mathrm{eV} \cdot n_{x}=n_{y}=20$ are the number of the molecules in $x$ and $y$ directions. This set of parameters will give a polaron formation energy of $E_{p}=-0.03 \mathrm{eV}$. ${ }^{1} R_{i, j}$ is a general symbol which can be
either $u_{i, j}$ or $v_{i, j}$.

${ }^{2}$ When an electron or a hole is introduced to the system. 
values of transfer integral $\left(J_{0}^{x, y}>0\right)$. In Holstein model, the polaron is defined as the ground state of a lattice with additional charge. The solutions can thus cover a continuous transition from a small polaron begin localized on a single site $\left(E_{p} \gg J\right)$ to a large polaron extended over several sites $\left(E_{p} \sim J\right)[131,33]$. The inclusion of the nonlocal Peierls electron-phonon interaction results in a slightly less localized but more stable polaron [132]. We can see from our calculations that taking both local and nonlocal electron-phonon interactions not only have an impact on the polaron formation energy and thus its stability but it also affects its shape. A more detailed discussion can be found in Paper I.

\subsection{Polaron Dynamics in a Two-Dimensional System}

To study the polaron dynamics, we have to solve Eqs. 4.5-4.7 and the time dependent Schrödinegr equation, Eq. 2.16, simultaneously. For this purpose, we use the ordinary differential equation (ODE) solver as implemented in MATLAB.

We recall the quantum adiabatic theorem, if we start in the nth eigenstate of the system, and the Hamiltonian is changed sufficiently slowly, the system remains in the nth eigenstate of the instantaneous Hamiltonian [133]. In our calculations, we consider an adiabatic approximation and the wavefunction of the charge is assumed to be a single eigenstate of the total Hamiltonian. ${ }^{3}$ We then study how this eigenstate evolves under the effect of an external electric field, temperature and disorder.

Figure 4.4 displays the results for the polaron dynamics calculations of a one dimensional system of 20 sites using the same parameter set as in Fig. 4.3. Starting with a stable polaron solution, an electric field is applied. As it is seen the polaron remains localized and immobile until about $250 \mathrm{fs}$. It is only after this time that it starts moving with a constant velocity of $\sim 25 \AA / p s$. The calculations are done under the assumption of periodic boundary conditions meaning that when the polaron reaches to one end of the system during the simulation time, it will reappear in the other end.

The left panel (a) shows the charge density, the middle panel (b) is the intramolecular displacements and the right one shows the intermolecular displacements (c) dynamics, respectively. Understanding how the polaron is transported in the system is of crucial importance. From the charge density dynamics, Fig. 4.4 (a), it can be seen that in the beginning of the simulation the polaron is localized on two sites. When the electric field is applied, the polaron starts to move. The transport can be described as an adiabatic motion in which at an instant of time, denoted as $t_{1}$, the particle goes under a transformation from being localized on a single site to being shared between two molecules at $t_{2}$. The polaron, thereafter, drifts and becomes localized on the next molecule at $t_{3}$. This process is shown schematically in Fig. 4.5. ${ }^{3}$ We have considered the lowest LUMO
in our simulations. 


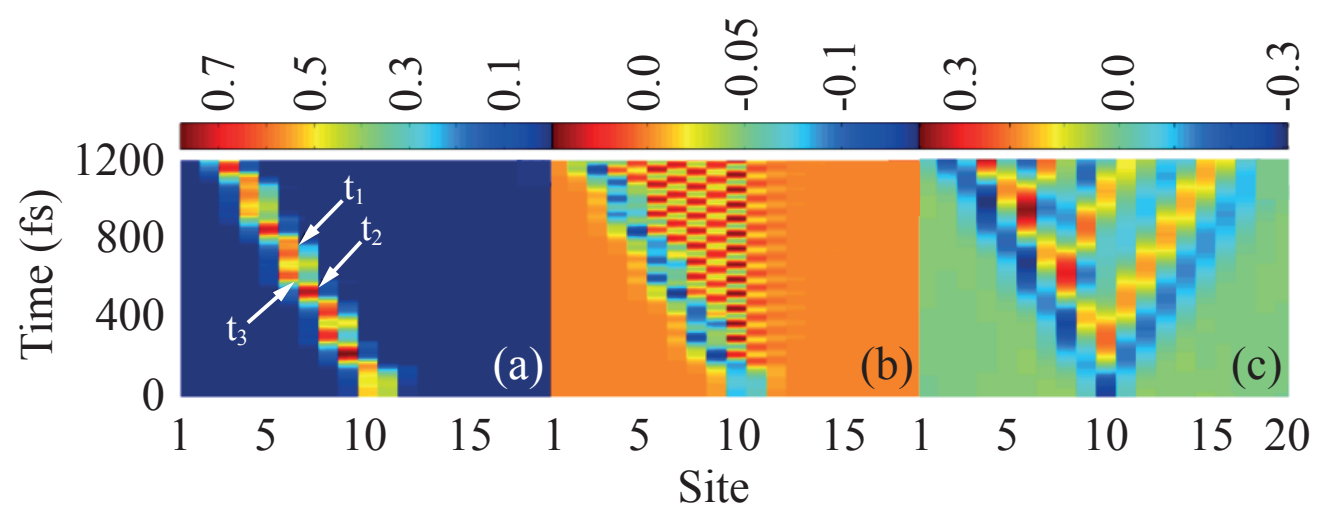

The potential energy, Eq. 4.9, is divided into two parts, one part comes from the intramolecular vibrations $E_{\text {pot }}^{\text {intra }}$ and the other from the intermolecular vibrations $E_{\text {pot }}^{\text {inter }}$. Figure 4.4 (b) demonstrates the time evolution of the local vibrations $u_{i} \mathrm{~s} .{ }^{4}$ We can see that these vibrations exhibit an oscillatory behavior with a frequency of $\sqrt{\frac{K_{1}}{m}}$. The intermolecular vibrations, Fig. 4.4 (c), also show an oscillatory behavior with the frequency obtained from $\sqrt{\frac{K_{2}}{M}}$. However, the nature of these vibrations is quite different from that of the intramolecular ones. In fact, the intermolecular distances, $\left(v_{i+1}-v_{i}\right) \mathrm{s}$, form traveling waves in the system which are moving with approximately the same velocity as the polaron but in the opposite direction. To be more precise, when the polaron is located on site $i$, the bond length between the neighboring site $i-1$ and site $i$ gets contracted (blue color in panels (b) and (c)) which in turn results in an expanded bond length between sites $i$ and $i+1$ (red color in panels (b) and (c)). Moreover, we can clearly see that both local and nonlocal vibrations follow the dynamics of the charge density proving that the moving polaron possesses the nature of a Holstein-Peierls polaron. More details about the polaron transport process in a $2 \mathrm{D}$ system can be found in Paper II.

We have performed numerous calculations using different parameter sets. Most importantly the effect of the local $(A)$ and nonlocal $(\alpha)$ electron-phonon couplings on polaron dynamics is studied. Fixing the values of $K_{1}, K_{2}, m$ and $M$, we study the effect of the external electric field strength, the transfer integral and coupling constants individually. We found that for values of local electron-phonon coupling constant $1.2<A<1.7 \mathrm{eV} / \AA$, the polaron remains localized and moves with a constant velocity. These values correspond to the polaron formation energy of 25 to $44 \mathrm{meV}$. For each value of $A<$ $1.2 \mathrm{eV} / \AA$, the polaron is unstable and delocalizes into a band state upon applying the electric field. For intramolecular electron-phonon coupling values of $A>1.7 \mathrm{eV} / \AA$, the polaron is immobile. The same

Figure 4.4: Polaron dynamics in a one dimensional Holstein-Peierls system: (a) molecular charge density, (b) intramolecular displacements $u_{i}$, and (c) intermolecular bond length $\left(v_{i+1}-v_{i}\right)$. The parameter set used for this calculation is the same as Fig. 4.3. The external electric field has a strength of $E_{0}=2.0 \mathrm{mV} / \AA$.

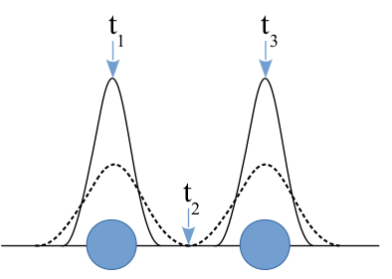

Figure 4.5: Schematic representation of adiabatic polaron transport process.

${ }^{4}$ Note that this is a one dimensional system and therefore we only have one index $i$ and only one direction $x$. 
behavior is observed for the values of intermolecular electron-phonon coupling constant $0.5<\alpha \leq 0.6 \mathrm{eV} / \AA$ for which we will have a dynamically stable polaron. The polaron formation energies, in this case, lie in a slightly higher range between 35 to $64 \mathrm{meV}$. These values of polaron formation energy suggests that increasing $\alpha$ results in a spatially more extended polaron whereas increasing $A$ makes the polaron to be more localized.

Varying the transfer integral values, $J_{i, j}^{x, y}$, we found that for the values in the range of 40 to $80 \mathrm{meV}$, the polaron is stable. These values correspond to the polaron formation energies of 44 to $80 \mathrm{meV}$. If $J_{i, j}^{x, y}<40 \mathrm{meV}$, the polaron will be immobile and if $J_{i, j}^{x, y}>80 \mathrm{meV}$, we will have a band state.

When the electric field strength is increased, the driving force on the polaron becomes larger. If this excess energy exceeds a critical value, the polaron will dissociate. An example of such behavior is shown in Fig. 4.6. Using the same parameter set as in Fig. 4.4, only the field strength is changed. We observe that for this $1 \mathrm{D}$ system with these set of parameters, the polaron destabilizes into a band state at $E_{0}=7.0$ $\mathrm{mV} / \AA ̊$, Fig. 4.6 (d).

For a two dimensional system, however, we found that the highest limit of the field strength for which the polaron is stable and mobile is $3.8 \mathrm{mv} / \AA$. For more details see Paper II.

\subsubsection{Polaron Dynamics in the Presence of Disorder}

Thermal vibrations, introducing impurities into the system either willingly by doping or unwillingly when defects are present in the system, can cause the system to deviate from its perfect crystal structure; i.e. it is disordered. The very first model to deal with disorder in the lattice was introduced by P. W. Anderson[134] in which the on-site energies are randomly distributed in a box of the width $W$ with equal probabilities $\varepsilon_{i j} \in\left(-\frac{W}{2}, \frac{W}{2}\right)$. In his study, Anderson showed once the disorder exceeds than a critical value of $\left(\frac{W}{B}\right)_{\text {critical }}, 5$ the solutions of the Schrödinger equation are not the Bloch extended states anymore rather become spatially localized. The charge, therefore, transports from one site to the next through exchanging energy with phonons in the lattice. However, the transition between extended and localized states has only been observed in three dimensional lattices. In lower dimensions, any non-zero value of disorder results in the localization of the charge. More detailed description of what happens in a two dimensional molecular lattice is given in Paper II.

\subsubsection{The Impact of the Temperature on Polaron Dynamics}

Depending on how high the temperature is, the transport process is classified into different regimes such as band transport, tunneling, temperature activated adiabatic transport and nonadiabatic transport. A significant insight into temperature effect on polaron transport can be traced back to Holstein's seminal work [33] in which the temperature dependence of the mobility as a function of local electron-

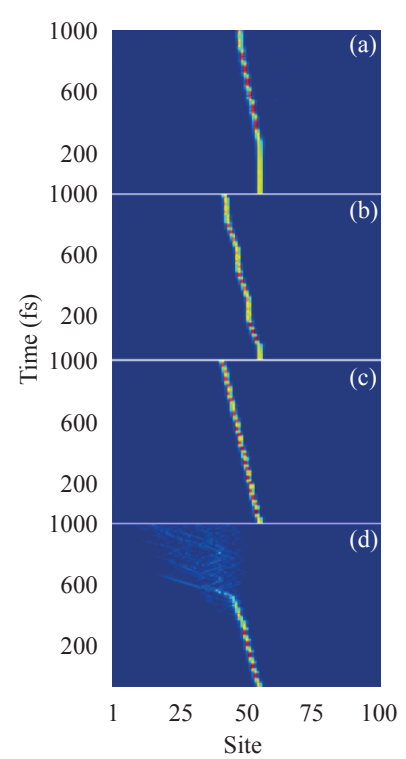

Figure 4.6: Polaron charge density dynamics of a one dimensional system as a function of electric field strength: (a) $E_{0}=0.5 \mathrm{mV} / \AA$, (b) $E_{0}=1.0 \mathrm{mV} / \AA$, (c) $E_{0}=3.0 \mathrm{mV} / \AA ̊$ And (d) $E_{0}=7.0$ $\mathrm{mV} / \AA$. The parameters are the same as in Fig. 4.3.

${ }^{5} \mathrm{~B}$ is the bandwidth. 
phonon coupling constant ( $g$ in Eq. 2.9) is predicted, Fig. 4.7. For weak electron-phonon couplings $\left(g^{2} \ll 1\right)$, the mobility shows a $\mu \sim T^{-n}(n>0)$ behavior and tunneling is the dominant way of transport. In the case of strong electron-phonon coupling $\left(g^{2} \gg\right.$ $1)$, three distinct temperature dependence of mobility is observable: (i) band-like transport at low temperatures, (ii) as the temperature increases, the hopping term becomes dominant and (iii) at very high temperatures, the thermal energy becomes large enough resulting in polaron dissociation. Thermal phonons scatter the residual electron and hence the mobility decreases again as the temperature increases. Despite the qualitative agreement between experimental data and what is obtained from solely Holstein theory, the charge transport mechanism in organic molecular crystals remains debatable and is not fully described. A way to handle this problem is given in Paper II in which we employ an SSH Hamiltonian to also include the nonlocal electron-phonon coupling in our calculations. In our studies, the temperature effect enters the simulation as a thermal random force, $R_{n}(t)[135,136,137]$ with zero mean value $\left\langle R_{n}(t)\right\rangle=0$ and the variances

$$
<R_{i j}^{i n t r a}(t) R_{i^{\prime} j^{\prime}}^{i n t r a}\left(t^{\prime}\right)>=2 k_{B} T m \lambda \delta_{i j ; i^{\prime} j^{\prime}}\left(t-t^{\prime}\right),
$$

and

$$
<R_{i j}^{\text {inter }}(t) R_{i^{\prime} j^{\prime}}^{\text {inter }}\left(t^{\prime}\right)>=2 k_{B} T M \lambda \delta_{i j ; i^{\prime} j^{\prime}}\left(t-t^{\prime}\right),
$$

for intra and intermolecular parts, respectively. $\lambda$ is a damping factor used to control the temperature variations. For a one dimensional system, thus, the lattice dynamics is governed by

$$
m \ddot{u}_{i}=-K_{1} u_{i}-A \rho_{i i}(t)-m \lambda \dot{u}_{i}(t)+R_{i}^{\text {intra }}(t),
$$

and

$$
\begin{aligned}
M \ddot{v}_{i} & =-K_{2}\left(v_{i}-v_{i+1}-v_{i-1}\right) \\
& -2 \alpha e^{i \gamma \Lambda(t)}\left(\rho_{i, i-1}(t)-\rho_{i+1, i}(t)\right) \\
& -M \lambda \dot{v}_{i}(t)+R_{i}^{\text {inter }}(t)
\end{aligned}
$$

These equations are no longer ordinary differential equations (ODE) but rather stochastical differential equations (SDE). Therefore, finding the right integrator for solving them is very important. One way is to use the Langevin dynamics. In our calculations, we employ the Brünger-Brooks-Karplus integrator (BBK) $[138,139]$ explained in more details in Appendix B.

The time evolution of the wave function is obtained by solving TDSE, Eq. 2.16. The solution of TDSE at each instant of time can be expressed as [83]

$$
\psi(n, t+\Delta t)=\sum_{l}\left[\sum_{m} \phi_{l}^{*}(m) \psi(m, t)\right] e^{-\left(i \varepsilon_{l} \Delta t / \hbar\right)} \phi_{l}(n),
$$

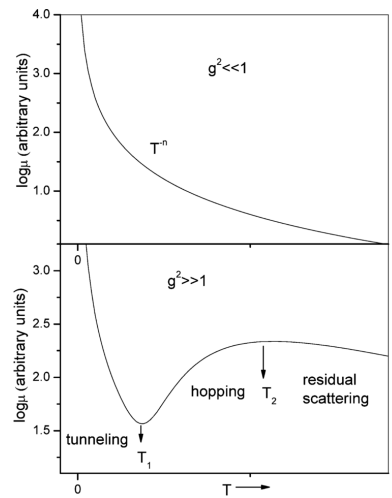

Figure 4.7: Mobility as a function of temperature as predicted by Holstein model for (a) weak and (b) strong local electronphonon coupling constants. Reprinted from Ref. [27]. 


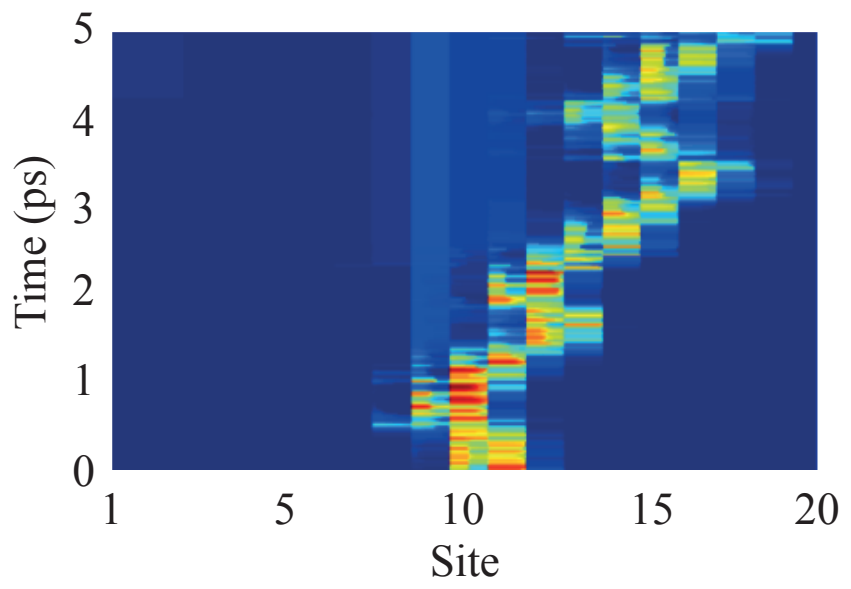

where $\phi_{l}(m)$ and $\varepsilon_{l}$ are the instantaneous eigenfunctions and eigenvalues of the electronic Hamiltonian $H_{e l}$ at time $t$, respectively. Solving Eqs. 4.17-4.19 simultaneously, we are able to study the polaron dynamics at different temperatures.

Starting with a zero-temperature polaronic ground state, obtained from RPROP, we perform a series of calculations on a $1 \mathrm{D}$ system in the temperature range of 50 to $300 \mathrm{~K}$ for a time period of 5 ps. The electric field is set to zero so that there is no external force to shove and trigger the polaron to move. Figure 4.8 demonstrates the polaron dynamics of a $1 \mathrm{D}$ Holstein-Peierls system at $\mathrm{T}=\mathbf{2 0 0} \mathrm{K}$. It is seen that the polaron is localized and has a diffusive motion due to the fluctuations imposed by temperature.

The total vibration energy of the lattice can be calculated from $\sum_{i}\left(m \dot{u}_{i}^{2} / 2+M \dot{v}_{i}^{2} / 2\right)$. At thermal equilibrium, this energy is equal to the inner energy of the lattice $\sum N k_{B} T / 2 .{ }^{11}$ As the temperature increases, the random forces, Eqs. 4.15-4.16, also increase. The larger the forces, the higher the amplitude of the lattice vibrations and the larger vibrational energy which in turn results in polaron dissociation. In fact, as in the case of the electric field, there also exist a critical temperature $T_{\text {critical }}$ above which the initial localized polaron will no longer be localized but rather destabilizes and dissociates into a band state, see Fig. 4.9. In our calculations for a one dimensional system, this critical temperature is found to be around $250 \mathrm{~K}$.

Note that even at temperatures higher than $T_{\text {critical }}$, depending on the magnitude of the temperature, it will takes a while for the localized polaron to evolve into a delocalized state. As the temperature increases, this time becomes shorter.

My studies are focused on the properties of the polaron in a two dimensional system. The role of the dimensionality on the stability of polarons is studied by Kalosakas et al. [140]. They have used the semiclassical Holstein model in one, two and three dimensions to
Figure 4.8: Molecular charge density of a one dimensional Holstein-Peierls system at $\mathrm{T}=200 \mathrm{~K}$ with $E_{0}=0.0 \mathrm{mV} / \AA ̊$ and $\lambda=$ $10^{2} \mathrm{eVfs} / \AA$. The other parameters are the as same as the ones used in Fig. 4.3.

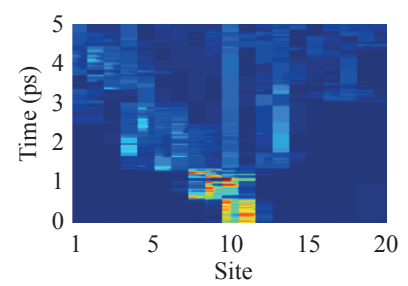

Figure 4.9: Molecular charge density of a one dimensional Holstein-Peierls system at $\mathrm{T}=300 \mathrm{~K}$. The parameters are as the same as Fig. 4.8.

${ }^{11} N \equiv$ degrees of freedom. 
study the energy and the existence regimes of the polaron state. Using normal-mode analysis, they have also investigated the small oscillations of the polaron, their stabilities. In their normal-mode analysis they found a pinning-breathing mode crossing in the one dimensional system in small to large polaron transition region which was not observed in higher dimensions. It is possible to derive scaling relations that are connecting the polaron self-energy, the effective mass and the mobility of a two dimensional system to their counterparts in a three dimensional system. Peeters and Devreese [72] have derived several of these scaling relations namely

$$
E_{2 D}(\alpha)=\frac{2}{3} E_{3 D}\left(\frac{3 \pi}{4} \alpha\right),
$$

and

$$
\mu_{2 D}(\alpha)=\mu_{3 D}\left(\frac{3 \pi}{4} \alpha\right) .
$$

Considering the role of dimensionality in the studies of polaron stability and its dynamics is therefore a necessity. Therefore, one needs to go to three dimensions in order to mimic realistic systems. In what follows, I will continue this chapter with the results and discussion of my second project on the electron-lattice interaction in magnetic crystals.

\subsection{Role of $\mathrm{N}$ defects in Paramagnetic $\mathrm{CrN}$}

Being an industrially important material, $\mathrm{CrN}$ is studied quite extensively both experimentally and theoretically. However, despite the existence of defects, in particular point defects such as $\mathrm{N}$ vacancies $\left(V_{N}\right)$ and interstitials $\left(I_{N}\right)$ in experiments, almost all theoretical investigations of the properties of $\mathrm{CrN}$-based materials are based on the assumption of a stoichiometric nitrogen sublattice. Beside that the existence of defects the electronic structure of $\mathrm{CrN}$ specially in its paramagnetic phase at finite temperatures is affected due to the temperature-induced vibrations and the magneto-lattice interactions. Within DLM-MD, we have the possibility to study the paramagnetic materials with the inclusion of both magnetic disorder and lattice vibrations with and without the presence of defects. In this project, I have used DLM-MSM and DLM-MD to study the formation energies and also the electronic structure of PM CrN considering the impact from the presence of $\mathrm{N}$ vacancies and $\mathrm{N}$ interstitials.

The formation energy of a single $\mathrm{N}$ defect is expressed as

$$
E_{F}=E_{d e f}-E_{0}+\frac{x}{2} E_{N_{2}}
$$

where $E_{0}$ is the total energy of the defect-free system, $E_{\text {def }}$ is the total energy of the system with a defect. $E_{N_{2}}$ is the energy of the $N_{2}$ molecule in vacuum and $x$ is +1 or -1 for $\mathrm{N}$ vacancy and $\mathrm{N}$ interstitial, respectively.

A $I_{N}$ can sit in different positions in the supercell namely in the tetragonal position, along the $<110>$ direction or the $<111>$

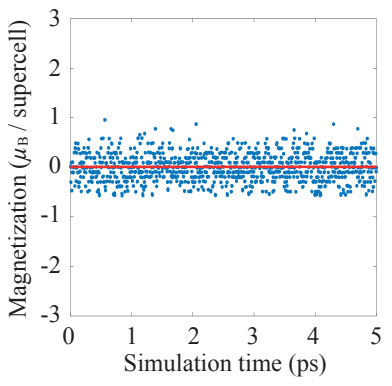

Figure 4.10: Time evolution of the net magnetization per supercell of $\mathrm{CrN}$ in the presence of a single $\mathrm{N}$ vacancy at $\mathrm{T}=1200 \mathrm{~K}$ shown by blue circles. The DLM spin configuration is changed every $\Delta t_{s f}=5 \mathrm{fs}$. The red solid line shows the average value of the net magnetization during the simulation. 
Table 4.1: Relative stability of nitrogen point defects in paramagnetic CrN obtained from Static DLM and DLM-MD calculations at different temperatures. Reprinted from Ref. [141].

\begin{tabular}{|c|c|c|c|c|c|c|c|c|}
\hline \multirow{2}{*}{ Defect } & \multirow{2}{*}{ Geometry } & \multirow{2}{*}{$E_{F}^{a}(\mathrm{eV} /$ defect $)$ Static DLM } & \multicolumn{6}{|c|}{$E_{F}(\mathrm{eV} /$ defect $)$ DLM-MD } \\
\hline & & & $300 \mathrm{~K}$ & $600 \mathrm{~K}$ & $700 \mathrm{~K}$ & $900 \mathrm{~K}$ & $1000 \mathrm{~K}$ & $1200 \mathrm{~K}$ \\
\hline$V_{N}$ & & 2.28 & 2.37 & 2.40 & 2.38 & 2.32 & 2.37 & 2.30 \\
\hline$I_{N}^{\text {tet }}$ & Tetrahedral & Metastable $^{b}$ & $c$ & & & & & \\
\hline$I_{N}^{110}$ & Split-bond $\langle 110\rangle$ & 3.84 & $c$ & & & & & \\
\hline$I_{N}^{111}$ & Split-bond $\langle 111\rangle$ & $3 \cdot 77$ & 3.80 & 3.84 & 3.87 & 3.87 & 3.97 & 3.88 \\
\hline
\end{tabular}

$a$ The energies are obtained from DLM-MSM.

${ }^{b}$ During our MSM calculations we noticed that an interstitial in the tetrahedral configuration would either remains in the tetrahedral position or go into the split-bond $I_{N}^{111}$ configuration (See the text).

${ }^{c}$ DLM-MD calculations at various temperatures show that the tetrahedral and the split-bond $\langle 110\rangle$ configurations are unstable. Instead the N-N pair positions itself along the $\langle 111\rangle$ direction.

direction. The obtained formation energies from both Static DLMMSM calculations and DLM-MD is summarized in Tab. 4.4. The N vacancy formation energy shows a weak temperature dependence as the difference between the results from static MSM-DLM and DLMMD calculations is very small. In this case, one can trust the static calculations to decide if the structure is stable. In contrast, in the $\mathrm{N}$ interstitial calculations a very different behavior is observed. The only stable configuration at finite temperatures is the $<111>$ split-bond and static calculations are not reliable enough to decide over the stability of the structure. This indicates the importance of treating the magnetic excitations and lattice vibrations simultaneously for studies of high temperature paramagnets, as it is done within our method. In its PM phase, the defect-free $\mathrm{CrN}$ is a semiconductor with a narrow insulating gap which is closed in the presence of $\mathrm{N}$ defects, even at zero temperature, resulting in metallic behavior. We can conclude that both $\mathrm{N}$ vacancy and $\mathrm{N}$ interstitial act as donors adding electrons to the system.

As mentioned in Sec. 3.3.2, to make sure the system remains in the DLM state, the net magnetization of the system should be checked. Fig. 4.10 shows the evolution of the net magnetization in the presence of a $\mathrm{N}$ vacancy at $\mathrm{T}=1200 \mathrm{~K}$.

We see that the net magnetic moment shows small fluctuations during the simulation but in total it averages to almost zero. More details about this project can be read in Paper III.

\subsection{Vibrational free energy and phase stability of $\mathrm{CrN}$}

The Helmholtz free energy can be expressed as

$$
F=<U_{D L M-M D}>+<E_{k}>-T S_{v i b},
$$

where the average potential energy $<U_{D L M-M D}>$ and the average kinetic energy $\left\langle E_{k}>\right.$ are extracted from DLM-MD calculations. However, the vibrational entropy $S_{v i b}$ is not explicitly accessible within DLM-MD. As mentioned earlier in Sec. 3.4, TDEP provides us with a 


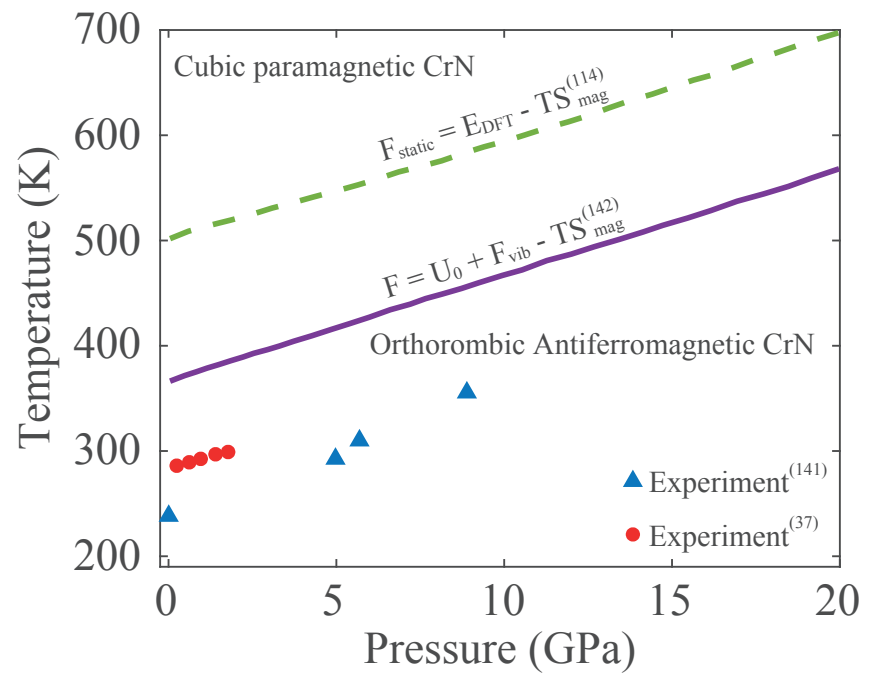

way to obtain the contribution of the full free vibrational energy in Eq. 3.2 including the implicitly calculated vibrational entropy. Alling et al. has recently demonstrated that including the magnetic entropy via Eq. 3.31 in the calculations of the PM CrN influences the Gibbs free energy to a large extent [115]. In their study using SQS to model the magnetic disorder, they predicted a phase transition temperature between the high temperature PM CrN phase and the low temperature AFM phase to be $498 \mathrm{~K}$. However, they have not taken the vibratinal contribution to the free energy into account.

In this project, we have used a combination of DLM-MD and TDEP to study the phase stability of paramagnetic $\mathrm{CrN}$. To model the antiferromagnetic phase of $\mathrm{CrN}$, we used the spin polarized ab initio molecular dynamics (AIMD) in cobination with TDEP. The obtained phonon dispersions show both AFM and PM phases are stable. We found that including the vibrational contribution in the free energy calculations of both phases results in a shift in the transition temperature to a lower value of $381 \mathrm{~K}$, Fig. 4.11. Although the obtained value for the phase transition temperature is still $\sim 100 \mathrm{~K}$ higher than the experimental value $\sim 286 \mathrm{~K}$, this study gives a first approximation for simultaneously including the magnetic and full vibrational contributions to the free energy. More details can be found in Paper V.

\subsection{Temperature-Dependent Elastic Properties of $\mathrm{CrN}$}

One important property to be studied in materials specially with industrial applications is the hardness. However, quantifying the hardness theoretically is quite difficult as it depends on a large set of underlying properties of the material such as its single crystal elastic constants, its pollycrystalline elastic moduli, its microstructure
Figure 4.11: Pressure-temperature phase diagram of $\mathrm{CrN}$ calculated by means of DLM-MD-TDEP method using the Helmholtz free energy as in Eq. 3.29 shown with the purple solid line from Ref. [143]. Experimental phase diagrams are taken from Ref. [37] (red circles) and Ref. [142] (blue triangles). The green dashed line corresponds to the phase diagram obtained from static calculations in Ref. [115]. Regenerated from Ref. [143]. 


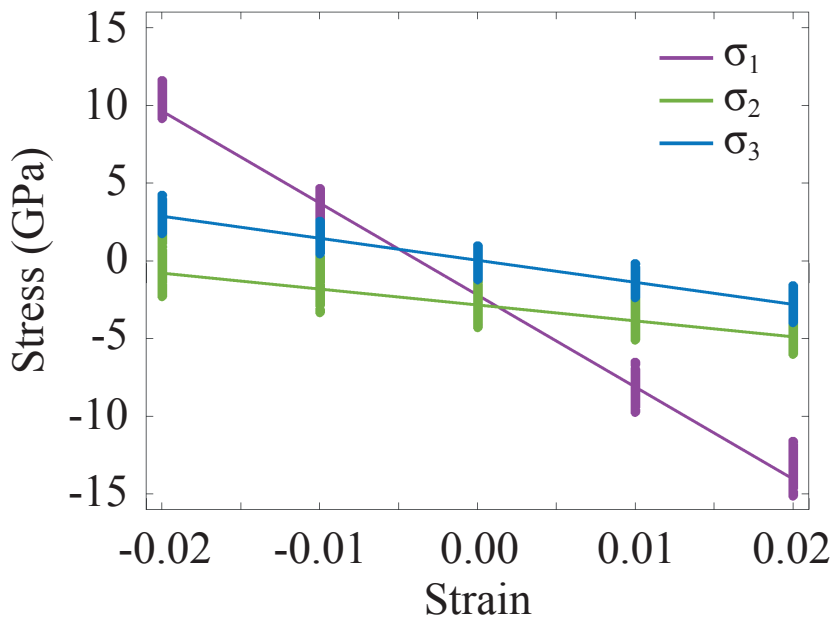

and many more. On the other hand, in magnetic materials above their magnetic transition temperature, in their paramagnetic state, it is shown that the elastic properties are largely affected by the existence of the local magnetic moments[37, 38]. Thus a possibility of predicting the temperature-dependent elastic properties of this class of materials theoretically is of high interest. The DLM-MD method, introduced in Sec. 3.3, provides us with this opportunity. Choosing the paramagnetic cubic $\mathrm{CrN}$ as our model system, we studied the temperature-dependence of its elastic properties. We have also used the SIFC-TDEP method, discussed in Sec. 3.4, to calculate the same properties. Details of the calculations can be read in Paper IV.

In summary, at each temperature, a set of deformations ${ }^{6}$ is applied to the supercell and the DLM-MD calculations are run. The deformation matrix for a cubic system is described as [144, 145]

$$
\epsilon=\left(\begin{array}{ccc}
\eta & \frac{\eta}{2} & 0 \\
\frac{\eta}{2} & 0 & 0 \\
0 & 0 & 0
\end{array}\right) .
$$

The resulting stresses $\sigma$ versus $\eta$ values for $300 \mathrm{~K}$ is shown in Fig. 4.12. The points are the stress values at each time step of the simulation and their verical spread show the variation of the stresses during the DLM-MD run. We can then fit a line to these points (solid lines in Fig. 4.12) and obtain the single crystal elastic constant values via the following relations. ${ }^{7}$

$$
\frac{\mathrm{d} \sigma_{1}}{\mathrm{~d} \eta}=C_{11} \quad, \quad \frac{\mathrm{d} \sigma_{2}}{\mathrm{~d} \eta}=C_{12} \quad, \quad \frac{\mathrm{d} \sigma_{3}}{\mathrm{~d} \eta}=C_{44} .
$$

I should also mention that Eq. 4.25 give the external stresses but Fig. 4.12 shows the internal stresses directly obtained from VASP calculations. Therefore, there is a difference in the sign of the derivatives.
Figure 4.12: The stress versus strain values for paramagnetic cubic $\mathrm{CrN}$ at $\mathrm{T}=300 \mathrm{~K}$. The points are the instantaneous stresses from each molecular dynamics time step. The vertical spread of the points can be seen as the variation of the stresses during the whole DLM-MD simulation. The solid lines are the linear fits to these points.

${ }^{6}$ for $\eta \in\{-0.02,-0.01,0.00,0.01,0.02\}$ in the deformation matrix $\epsilon$

${ }^{7}$ For a cubic system due to the symmetry, we only have three independent elastic constants. 
We observed that the elastic constants decrease almost linearly with temperature. Our calculated Young's modulus $E=433 \mathrm{GPa}$ is in good agreement with the experimental value [146] $E=400 \mathrm{GPa}$ at room temperature and decreases by about $14 \%$ at $1200 \mathrm{~K}$.

Figure 4.13 shows the experimental as well as the theoretical temperaturedependent lattice constant of the cubic CrN. In our calculations, we have adopted the experimental values. However, as it is apparent, both data sets are in very good agreement with each other and therefore using the experimental values do not affect the accuracy of the calculations.

\subsection{The Electronic Structure of $\mathrm{NiO}$}

Despite all experimental and theoretical studies on $\mathrm{NiO}[147,148,149$, 150, 151, 152, 153, 154, 155, 61], its electronic structure has remained an ambiguity. The different very advanced theoretical methods or even the same method ${ }^{8}[153,61]$ result in different electronic spectrum. These inconsistencies calls for a benchmark study with a computationally much more robust and well-tested $\mathrm{LDA}+\mathrm{U}$ approach together with DLM method.

It is generally believed that the electronic structure of $\mathrm{NiO}$ is not affected by the magnetic phase transition from the low temperature AFM to the high temperature PM phase and hence the electronic spectrum of the PM phase is often compared to that of the experimental photo-emission data of the AFM phase [156, 152, 153, 61]. Our DLM-MSM calculations to get the electronic structure at $\mathrm{T}=\mathrm{o} \mathrm{K}$ show that even though this assumption is not far from reality, including

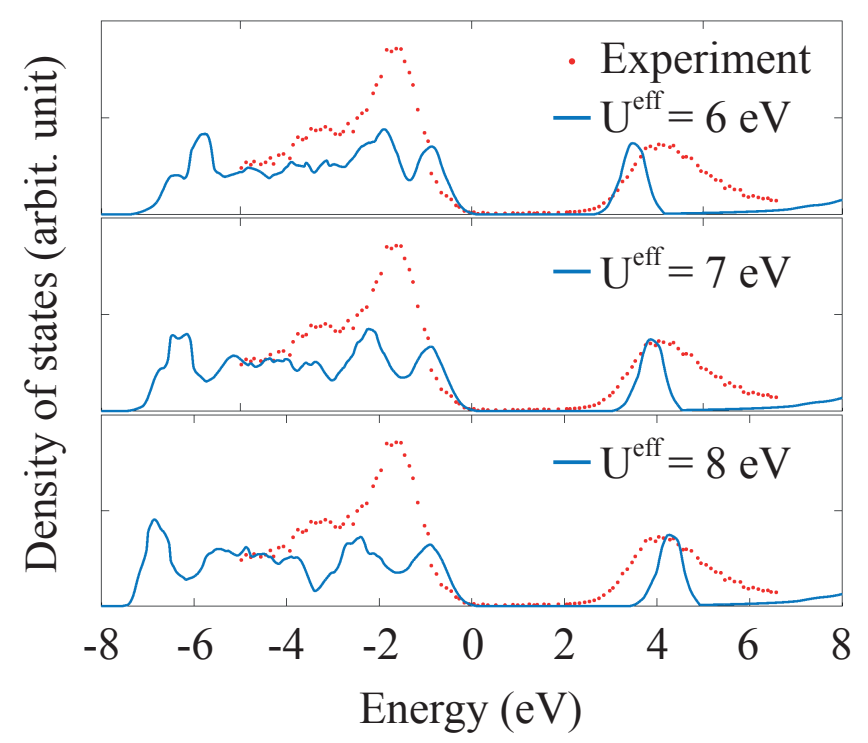

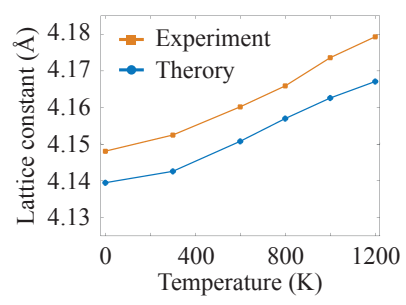

Figure 4.13: Temperature-dependent lattice constant of the cubic $\mathrm{CrN}$ from experiment[142] and theory.

${ }^{8}$ Depending on the input parameters such as the lattice constant or the Hubbard term $U$.

Figure 4.14: Zero Kelvin ( $\mathrm{T}=\mathrm{o} \mathrm{K})$ electronic structure of rhombohedral antiferromagnetic unit cell of $\mathrm{NiO}$ within $\mathrm{LDA}+\mathrm{U}$ approximation with different $U^{e f f}=U-J$ values for $J=1 \mathrm{eV}$ and experimental lattice parameter $\mathrm{a}=4.17 \AA$. The experimental photo-emission spectroscopy measurement from Ref. [148] is also shown for comparison with dotted line in all frames. The Brillouin zone is sampled via a Monkhorst-Pack $23 \times 23 \times 23 k$-point grid and the energy cut-off is set to $500 \mathrm{eV}$. The zero energy point corresponds to the top of the valence band, while the height of the peaks is adjusted to fit the experimental highenergy peak height. 


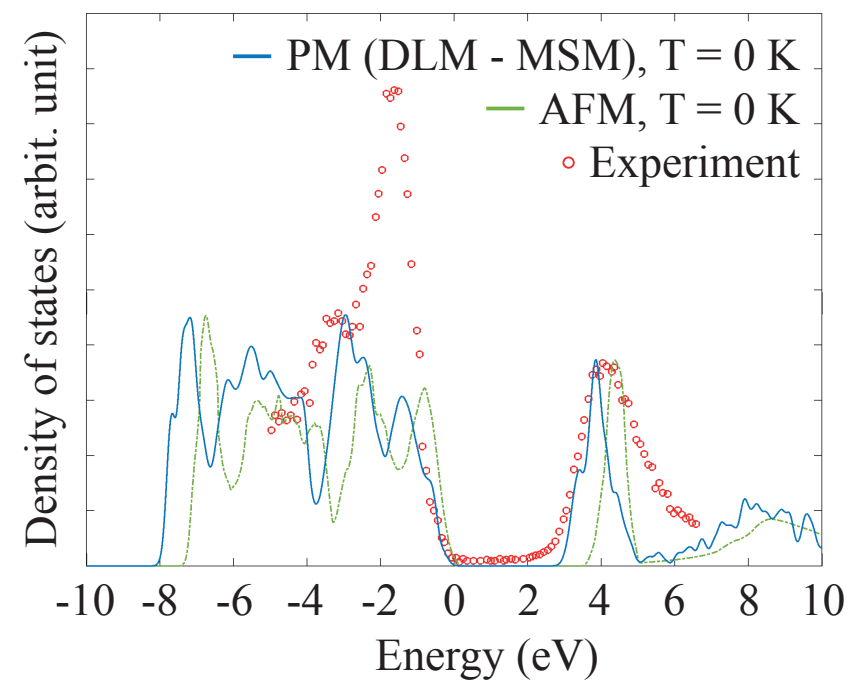

magnetic disorder in the PM phase calculations have an impact, although quite small, on the density of states. The band gap becomes smaller than that of the AFM phase, Fig. 4.15. We are, therefore, led to the question of what would happen at higher temperatures when the lattice dynamics is also added.

From our DLM-MD calculations, we can observe that small temperature variations do not affect the electronic structure to a large degree and hence one can rely on the static calculations in which the thermal expansion coefficient is included. However, as the temperature increases, the lattice dynamics affects the electronic structure to a large extent. The band gap becomes visibly smaller and the peaks in the density of states become broader. In fact, the change in the electronic spectrum due to explicit lattice vibrations becomes more apparent and therefore cannot be ignored in the calculations.
Figure 4.15: Zero kelvin $(\mathrm{T}=\mathrm{o} \mathrm{K})$ electronic structure of antiferromagnetic (AFM) $\mathrm{NiO}$ from experiment (red circles) and theory (green dot-dashed line) and paramagnetic (PM) NiO from DLMMSM calculations (blue solid line). The theoretical calculations are carried out using $\mathrm{LDA}+\mathrm{U}$ with $U^{e f f}=8 \mathrm{eV}$ and experimental lattice parameter $a=4.17 \AA$. The experimental data is adopted from Ref. [148]. The zero energy point corresponds to the top of the valence band, while the height of the peaks is adjusted to fit the experimental high-energy peak height. 



\section{5 \\ Conclusion and Outlook}

$\mathfrak{S}$ HE unifying theme within this thesis is numerical studies of the electron-lattice interactions. The electron-lattice interaction is considered and studied in two groups of materials, namely molecular crystals and magnetic materials, throughout this thesis.

The quasi-particle "polaron" is a result of the dynamic electron-lattice interaction. A common way to study polaron dynamics is via Holstein model. Considering only local electron-phonon interactions, Holstein Hamiltonian neglects the effects of non-local interactions. In my work, I have extended the Holstein Hamiltonian to include the non-local electron-phonon interactions. For this purpose, I have added the Peierls Hamiltonian on top of the Holstein Hamiltonian. Using Holstein-Peierls model, in paper I and II, I have shown that for the proper description of the system, both local and non-local electron-phonon interactions should be considered. I also found that taking the lattice contribution into account through Peierls model substantially increases the stability of the polaron in our two dimensional model system. The effect of external electric field and disorder on polaron motion is also studied. According to our results, a stable adiabatically mobile Holstein-Peierls polaron solution is obtained for only a limited region of the parameter space which in good agreement with interaction strengths expected in molecular crystals. The fact that the polaron solution is confined to a specific choice of parameter sets suggests that the polaron motion can be system-dependent, i.e. it is likely that in some molecular crystals the charge transport is better described within the band model or in some others via the non-adiabatic hopping process. In particular, disorder in the system further reduces the possibility of achieving a dynamically stable and mobile polaron solution.

Another group of materials in which I have studied the interplay between electrons and lattice vibrations are high temperature paramagnetic materials. The magnetic disorder of the paramagnetic state is considered within the disordered local moment model and the effect of finite temperature lattice vibrations is included via molecular dynamics simulations. Combining these two will be the DLM-MD method which is used to study various temperature-dependent properties such as defect formation energies (in particular nitrogen $(\mathrm{N})$ 
defects), phase stabilities and vibrational free energies, electronic structure and elastic properties of magnetic materials in their paramagnetic state. We have mainly focused on $\mathrm{CrN}$ and $\mathrm{NiO}$ as our model systems but the method is general and can be applied to study any other paramagnetic system given that the input parameters such as the Hubbard $U$ term should be tested and chosen carefully. Beside the convergence of the energy and the evolution of the net magnetization should be checked.

Using DLM-MD, in paper III, I study the formation energies of $\mathrm{N}$ vacancies and $\mathrm{N}$ interstitials in paramagnetic $\mathrm{CrN}$. I found that the $\mathrm{N}$ vacancy formation energy obtained from static DLM calculations is in very good agreement with the ones from DLM-MD and therefore, one can rely on the results from static calculations to decide over the stability of the structure. The situation is, however, very different in $\mathrm{N}$ interstitials as the formation energy from DLM-MD at room temperature reveals that the only stable $\mathrm{N}$ interstitial structure is the $<111>$ split-bond whereas the static calculations suggests that the other structures, tetrahedral and $\langle 110\rangle$ split-bond are meta-stable and stable, respectively. Cubic paramagnetic $\mathrm{CrN}$ is a semiconductor with a small band gap. The electronic structure calculations showed that in the presence of $\mathrm{N}$ defects, this gap is closed and the system will have metallic characteristics. We note that even though within DLM$\mathrm{MD}$, it is possible to implicitly include the effect of lattice vibrations, the vibrational entropy is not explicitly included in the simulations. In order to achieve this goal, we have combined the DLM-MD method with the temperature dependent effective potential (TDEP) method in paper V. This has enabled us to calculate the magnetic order-disorder transition temperature of $\mathrm{CrN}$ in a better agreement with experiment than static calculations. Phonon dispersion relation calculations also show that both ordered antiferromagnetic and disordered paramagnetic phases of $\mathrm{CrN}$ are dynamically stable. A necessary condition for dynamical stability of a structure is that it remains stable under small perturbations of its atomic positions. In paper IV, using stress-strain relation and running DLM-MD calculations, I calculated both single crystal and polycrystalline elastic constants of $\mathrm{CrN}$ as a function of temperature. The obtained elastic constants show a linear decrease with increasing temperature and the polycrystalline elastic constants deacrease by $14 \%$ between room temperature and $1200 \mathrm{~K}$. This strong temperature dependence of elastic properties suggests that the effect of temperature and lattice vibrations should be taken into account in the calculations of elastic constants of high temperature paramagnetic materials.

Last but not the least, I have studied the finite temperature electronic structure of paramagnetic NiO by means of DLM-MD in paper VI. Our obtained results suggest that the electronic structure of paramagnetic $\mathrm{NiO}$ is not strongly affected by lattice vibrations up to 1000 $\mathrm{K}$ and therefore static DLM calculations can be safely employed in simulations of the paramagnetic state of $\mathrm{NiO}$. However, at higher temperatures close to the melting temperature of $\mathrm{NiO}(\sim 2230 \mathrm{~K})$, 
vibrational effects become prominent, the band gap becomes visibly smaller and the peaks in the density of states become broader.

In summary, all of the above results demonstrate the importance of taking the lattice contribution into account in simulations of materials. Despite the fact that lattice vibrations might be considered to have a small effect on the material's properties, in my work I have shown the importance of taking them into account. In fact, the accuracy of any semi-classical model Hamiltonian in the description of a system, depends on how well the interaction of the electronic and lattice degrees of freedom is reflected through its interaction parameters. The results from the numerical calculations in this thesis demonstrate the reliability of the model Hamiltonians that I have harnessed plus the importance of electron-lattice interactions for the proper description of a material. 

Appendices 



\section{$A$ \\ Resilient Propagation (RPROP) Algorithm}

RPROP stand for resilient backpropagation which is first-order optimization algorithm created by Martin Riedmiller and Heinrich Braun in 1992[? ]. It is a very efficient technique which is based on the sign but not the magnitude of the partial derivative. In other words, it acts independently on each "weight" step based on local gradient information.

Verbalized, for each weight, if the last update was too big and has jumped over a local minimum, the partial derivative of the total error function $\frac{\partial E}{\partial \omega_{i j}}$ changes its sign. Thus, the update value $\Delta_{i j}$ will be multiplied by a factor of $\eta^{-}$, where $\eta^{-}<1$. On the other hand, if the derivative has the same sign, the update value will be increased by a factor of $\eta^{+}$, where $\eta^{+}>1$. The process is over when each weight is updated according to its update value and the total error function $E$ is minimized. $\eta^{+}$and $\eta^{-}$are empirically set to 1.2 and 0.5 , respectively. RPROP adaptation and learning process is expressed in the following MATLAB code fragment in which the max ( $\mathrm{min}$ ) operator delivers the minimum (maximum) of two numbers and the sign operator returns +1 for a positive argument, -1 for a negative argument and o otherwise.

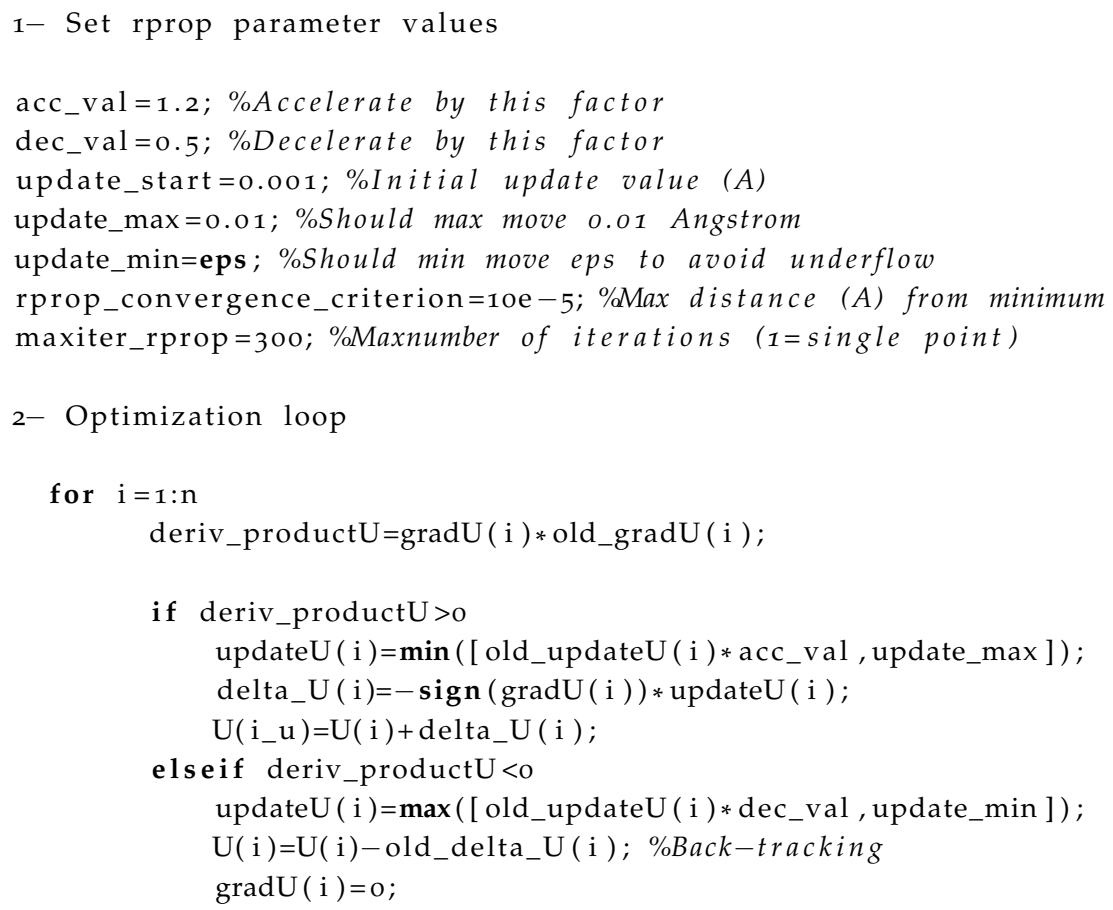


72 theoretical description of the electron-lattice interaction in molecular and magnetic crystals

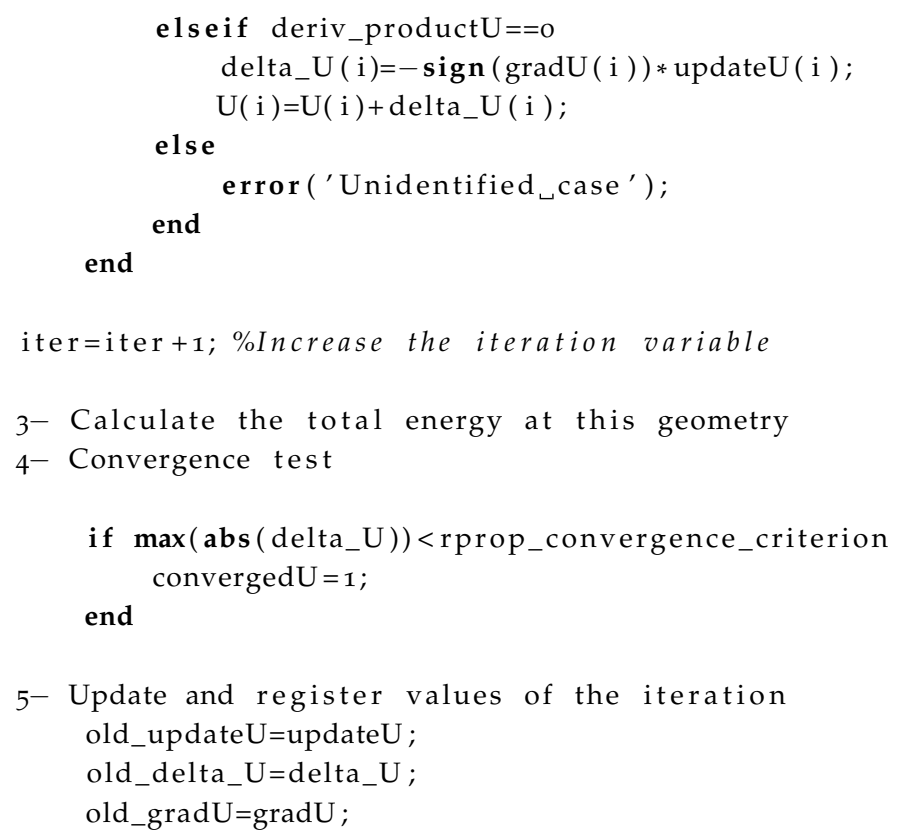

End of optimization loop 


\section{$B$}

\section{Brünger, Brooks and Karplu (BBK) Algorithm}

Brünger, Brooks and Karplus[138] gave a popular solution to Langevin equation which is known as BBK integrator. Having considered the Newtonian equations of motion, Eqs: 4.17-4.18, denoted by the term $F^{n}$, we choose the general letter $X$ to address both intra and intermolecular displacements. Consequently $\dot{X}$ and $\ddot{X}$ are the velocity and the accelerations, respectively. $M$ can be either of the intra or intermolecular masses. The algorithm for updating the velocities and positions are then expressed as

half a kick

$$
\dot{X}^{+\frac{1}{2}}=\left(1-\frac{1}{2} \lambda \Delta t\right) \dot{X}^{n}+\frac{1}{2 M}\left(F^{n}+R^{n}\right) \Delta t
$$

drift

$$
X^{n+1}=X^{n}+\Delta t \dot{X}^{n+\frac{1}{2}}
$$

half a kick

$$
\dot{X}^{n+1}=\frac{\dot{X}^{n+\frac{1}{2}}+\frac{1}{2 M}\left(F^{n+1}+R^{n+1}\right) \Delta t}{1+\frac{1}{2} \lambda \Delta t}
$$

where $R^{n}=\sqrt{\frac{2 \lambda k_{B} T}{\Delta t}} M^{1 / 2} Z^{n}$ with $Z^{n}$ being a vector of independent Gaussian random numbers of zero mean and variance 1 . $n$ is the counter of the time step $\Delta t$. Therefore, $n+\frac{1}{2}$ shows half of the time step, $t \rightarrow t+\frac{\Delta t}{2}$. 

6

\section{Bibliography}

[1] N. W. Ashcroft and D. Mermin. Solid state physics. Brooks/Cole, New York, 1976.

[2] E.O. Kane. Energy band structure in p-type germanium and silicon. J. Phys. Chem. Solids, 1(1-2):82-99, 1956.

[3] J. M. Luttinger and W Kohn. Motion of Electrons and Holes in Perturbed Periodic Fields. Phys. Rev., 97(4):869-883, feb 1955.

[4] Y. Toyozawa. Optical processes in solids. Cambridge University Press, Cambridge, United Kingdom, 2003 .

[5] J. B. Staunton, D. D. Johnson, and F. J. Pinski. Theory of compositional and magnetic correlations in alloys: Interpretation of a diffuse neutron-scattering experiment on an iron-vanadium single crystal. Phys. Rev. Lett., 65(10):1259-1262, sep 1990.

[6] J. B. Staunton, D. D. Johnson, and B. L. Gyorffy. Interaction between magnetic and compositional order in Ni-rich NicFe1-c alloys (invited). J. Appl. Phys., 61(8):3693, 1987.

[7] H. Hasegawa. Single-Site Functional-Integral Approach to Itinerant-Electron Ferromagnetism. J. Phys. Soc. Japan, 46(5):1504-1514, may 1979.

[8] H. Hasegawa. Single-Siet Spin Fluctuation Theory of Itinerant-Electron Systems with Narrow Bands. J. Phys. Soc. Japan, 49(1):178-188, jul 1980.

[9] J. Hubbard. Magnetism of iron. II. Phys. Rev. B, 20(11):4584-4595, dec 1979.

[10] J. Hubbard. The magnetism of iron. Phys. Rev. B, 19(5):2626-2636, mar 1979.

[11] J. Hubbard. Magnetism of nickel. Phys. Rev. B, 23(11):5974-5977, jun 1981.

[12] B. L. Gyorffy, A. J. Pindor, J. B. Staunton, G. M. Stocks, and H. Winter. A first-principles theory of ferromagnetic phase transitions in metals. J. Phys. F Met. Phys., 15(6):1337-1386, jun 1985.

[13] M. Berggren, O. Inganäs, G. Gustafsson, J. Rasmusson, M. R. Andersson, T. Hjertberg, and O. Wennerström. Light-emitting diodes with variable colours from polymer blends. Nature, 372(6505):444-446, dec 1994 .

[14] A. J. Heeger. Light emission from semiconducting polymers: Light-emitting diodes, light-emitting electrochemical cells, lasers and white light for the future. Solid State Commun., 107(11):673-679, 1998.

[15] J. H. Burroughes, D. D. C. Bradley, A. R. Brown, R. N. Marks, K. Mackay, R. H. Friend, P. L. Burns, and A. B. Holmes. Light-emitting diodes based on conjugated polymers. Nature, 347(6293):539-541, oct 1990.

[16] J. H. Burroughes, C. A. Jones, and R. H. Friend. New semiconductor device physics in polymer diodes and transistors. Nature, 335(6186):137-141, sep 1988.

[17] H. Sirringhaus, N. Tessler, and R. H. Friend. Integrated optoelectronic devices based on conjugated polymers. Science (80-. )., 280(5370):1741-1744, 1998. 
[18] M. E. Gershenson, V. Podzorov, and A. F. Morpurgo. Colloquium : Electronic transport in singlecrystal organic transistors. Rev. Mod. Phys., 78(3):973-989, sep 2006.

[19] M. Granström, K. Petritsch, A. C. Arias, A. Lux, M. R. Andersson, and R. H. Friend. Laminated fabrication of polymeric photovoltaic diodes. Nature, 395:257-260, 1998.

[20] S. E. Shaheen, C. J. Brabec, N. S. Sariciftci, F. Padinger, T. Fromherz, and J. C. Hummelen. 2.5\% Efficient Organic Plastic Solar Cells. Appl. Phys. Lett., 78(6):841-843, 2001.

[21] V. Andersson, K. Tvingstedt, and O. Inganäs. Optical modeling of a folded organic solar cell. J. Appl. Phys., 103(9), 2008.

[22] O. D. Jurchescu, J. Baas, and T. T. M. Palstra. Effect of impurities on the mobility of single crystal pentacene. Appl. Phys. Lett., 84(16):3061-3063, 2004.

[23] V. C. Sundar, J. Zaumseil, V. Podzorov, E. Menard, R. L. Willett, T. Someya, M. E. Gershenson, and J. A. Rogers. Elastomeric transistor stamps: reversible probing of charge transport in organic crystals. Science, 303(5664):1644-1646, mar 2004.

[24] I. N. Hulea, S. Fratini, H. Xie, C. L. Mulder, N. N. Iossad, G. Rastelli, S. Ciuchi, and A. F. Morpurgo. Tunable Fröhlich polarons in organic single-crystal transistors. Nat. mater., 5(12):982-6, dec 2006.

[25] N. Koch, A. Vollmer, I. Salzmann, B. Nickel, H. Weiss, and J. P. Rabe. Evidence for temperaturedependent electron band dispersion in pentacene. Phys. Rev. Lett., 96(15):10-13, 2006.

[26] N. Karl. Organic Semiconductors(Landolt-Börnstein Numerical Data and Functional Relationships in Science and Technology (New Series), Group III) vol 17i. pages 106-218. Springer, Berlin, 1985.

[27] V. Coropceanu, J. Cornil, F. da Silva, A. Demetrio, Y. Olivier, R. Silbey, and J. Bredas. Charge transport in organic semiconductors. Chem. Rev., 107(4):926-52, apr 2007.

[28] E. A. Silinsh and V. Capek. Organic Molecular Crystals: interaction, localization and transport phenomena. AIP Press, New York, 1994.

[29] Y. C. Cheng, R. J. Silbey, F. da Silva, J. P. Calbert, J. Cornil, and J. L. Bradas. Three-dimensional band structure and bandlike mobility in oligoacene single crystals: A theoretical investigation. J. Chem. Phys., 118(8):3764-3774, 2003.

[30] K. Hannewald, V. Stojanović, J. Schellekens, P. Bobbert, G. Kresse, and J. Hafner. Theory of polaron bandwidth narrowing in organic molecular crystals. Phys. Rev. B, 69(7):075211, feb 2004.

[31] K. Hummer and C. Ambrosch-Draxl. Electronic properties of oligoacenes from first principles. Phys. Rev. B - Condens. Matter Mater. Phys., 72(20):1-10, 2005.

[32] A. Troisi and G. Orlandi. Charge-transport regime of crystalline organic semiconductors: Diffusion limited by thermal off-diagonal electronic disorder. Phys. Rev. Lett., 96(8):1-4, 2006.

[33] T. Holstein. Studies of polaron motion,Part I\&II. Ann. Phys. (N. Y)., 8(3):325-389, nov 1959.

[34] G. D. Mahan. Many Particle Physics. Kluwer Academic Publishers, New York, 3rd edition, 2000.

[35] B. S. Baigrie. Electricity and Magnetism: A Historical Perspective. Greenwood Press, Westport, CT (USA), 2007.

[36] W. Gilbert. De Magnete (Translated by P. F. Mottelay). London, 1893.

[37] F. Rivadulla, M. Bañobre-López, C. X. Quintela, A. Piñeiro, V. Pardo, Daniel Baldomir, M. A. LópezQuintela, J. Rivas, C. A. Ramos, H. Salva, J. Zhou, and J. B. Goodenough. Reduction of the bulk modulus at high pressure in CrN. Nat. Mater., 8(12):947-951, dec 2009.

[38] B. Alling, T. Marten, and I. A. Abrikosov. Questionable collapse of the bulk modulus in CrN. Nat. Mater., 9(4):283-284, apr 2010. 
[39] M. Ekholm, H. Zapolsky, A. V. Ruban, I. Vernyhora, D. Ledue, and I. A. Abrikosov. Influence of the magnetic state on the chemical order-disorder transition temperature in Fe-Ni permalloy. Phys. Rev. Lett., 105(16):167208, 2010.

[40] P. Mohn. Magnetism in the solid state. Springer-Verlag, Berlin, Heidelberg, 2003.

[41] W. Heisenberg. Mehrkörperproblem und Resonanz in der Quantenmechanik. Zeitschr.f. Phys., 38:441, 1926.

[42] E. C. Stoner. Collective electron specific heat and spin paramagnetism in metals. Proc. Roy. Soc. A, 154:656-678, 1936.

[43] E. C. Stoner. Collective electron ferromagnetism. Proc. Roy. Soc. A, 165:372, 1938.

[44] B. L. Gyorffy, A. J. Pindor, J. B. Staunton, G. M. Stocks, and H. Winter. A first-principles theory of ferromagnetic phase transitions in metals. J. Phys. F Met. Phys., 15(6):1337-1386, jun 1985.

[45] T. Moriya. Recent progress in the theory of itinerant electron magnetism. J. Magn. Magn. Mater., 14, 1979 .

[46] T. Moriya. Spin fluctuations in itinerant electron magnetism. Springer-Verlag, Berlin, 1985.

[47] B. Alling, F. Körmann, B. Grabowski, A. Glensk, I. A. Abrikosov, and J. Neugebauer. Strong impact of lattice vibrations on electronic and magnetic properties of paramagnetic Fe revealed by disordered local moments molecular dynamics. Phys. Rev. B, 93:224411, 2016.

[48] O. Knotek, M. Atzor, A. Barimani, and F. Jungblut. Development of low temperature ternary coatings for high wear resistance. Surf. Coat. Technol., 42(1):21-28, 1990.

[49] B. Navinšek, P. Panjan, and I. Milošev. Industrial applications of CrN (PVD) coatings, deposited at high and low temperatures. Surf. Coatings Technol., 97(1-3):182-191, 1997.

[50] G. Berg, C. Friedrich, E. Broszeit, and C. Berger. Development of chromium nitride coatings substituting titanium nitride. Surf. Coatings Technol., 86-87(PART 1):184-191, 1996.

[51] A. E. Reiter, V. H. Derflinger, B. Hanselmann, T. Bachmann, and B. Sartory. Investigation of the properties of Al1-xCrxN coatings prepared by cathodic arc evaporation. Surf. Coatings Technol., 200(7):2114-2122, 2005.

[52] L. M. Corliss, N. Elliott, and J. M. Hastings. Antiferromagnetic Structure of CrN. Phys. Rev., 117(4):929-935, feb 1960.

[53] J. D. Browne, P. R. Liddell, R. Street, and T. Mills. An investigation of the antiferromagnetic transition of CrN. Phys. Status Solidi, 1(4):715-723, apr 1970.

[54] A. Filippetti, W. E. Pickett, and B. M. Klein. Competition between magnetic and structural transitions in CrN. Phys. Rev. B, 59(10):7043-7050, mar 1999.

[55] D. Gall, C. S. Shin, R. T. Haasch, I. Petrov, and J. E. Greene. Band gap in epitaxial NaCl-structure CrN(oo1) layers. J. Appl. Phys., 91(9):5882, 2002.

[56] R. Sanjinés, O. Banakh, C. Rojas, P. E. Schmid, and F. Lévy. Electronic properties of Cr1-xAlxN thin films deposited by reactive magnetron sputtering. Thin Solid Films, 420-421:312-317, dec 2002.

[57] P. Steneteg, B. Alling, and I. A. Abrikosov. Equation of state of paramagnetic CrN from ab initio molecular dynamics. Phys. Rev. B, 85(14):144404, apr 2012.

[58] L. F. Mattheiss. Electronic structure of the $3 d$ transition-metal monoxides. I. Energy-band results. Phys. Rev. B, 5(2):290-306, jan 1972.

[59] K. Terakura, T. Oguchi, A. R. Williams, and J. Kübler. Band theory of insulating transition-metal monoxides: Band-structure calculations. Phys. Rev. B, 3o(8):4734-4747, oct 1984.

[6o] K. Terakura, A. R. Williams, T. Oguchi, and J. Kübler. Transition-Metal Monoxides: Band or Mott Insulators. Phys. Rev. Lett., 52(20):1830-1833, may 1984. 
[61] S. K. Panda, B. Pal, S. Mandal, M. Gorgoi, S. Das, I. Sarkar, W. Drube, W. Sun, I. Di Marco, A. Lindblad, P. Thunström, A. Delin, O. Karis, Y. O. Kvashnin, M. van Schilfgaarde, O. Eriksson, and D. D. Sarma. High photon energy spectroscopy of $\mathrm{NiO}$ : Experiment and theory. Phys. Rev. B, 93(23):235138, jun 2016.

[62] L. D. Landau. Über die Bewegung der Elektronen in Kristalgitter. Phys. Z. Sowjetunion, 3:644-645, 1933 .

[63] S. I. Pekar. Local quantum states of an electron in an ideal ionic crystal. J. Phys., 10:341, 1946.

[64] S. I. Pekar. Research in Electron Theory of Crystals: Moscow (1951). English translation : US Atomic Energy Commission, Washington, 1963.

[65] Y. Toyozawa. On the Electronic Polaron State. Prog. Theor. Phys., 9(2):677-678, 1953.

[66] Y. Toyozawa. Theory of the Electronic Polaron and Ionization of a Trapped Electron by an Exciton. Prog. Theor. Phys., 12(4):421-442, 1954.

[67] H. Fröhlich. Electrons in lattice fields. Adv. Phys., 3(11):325-361, jul 1954.

[68] J. T. Devreese. Fröhlich Polaron concept. Lecture course including detailed theoretical derivations, 2010.

[69] C. G. Kuper and G. D. Whitfield. Polarons and Excitons. Oliver and Boyd Ltd., Edingburg and London, 1963.

[70] J. Appel. Polarons. In Solid State Phys., volume 21, pages 193-391. Academic Press, New York, 1968.

[71] T. K. Mitra, A. Chatterjee, and S. Mukhopadhyay. Polarons. Phys. Rep., 153(2-3):91-207, 1987.

[72] J. T. Devreese. Encyclopedia of Applied Physics. In Encycl. Appl. Phys., volume 14, pages 383-409. 1996.

[73] A. S. Mishchenko, N. V. Prokof'ev, A. Sakamoto, and B. V. Svistunov. Diagrammatic quantum Monte Carlo study of the Fröhlich polaron. Phys. Rev. B, 62(10):6317-6336, 2000.

[74] R. P. Feynman. Slow Electrons in Polar Crystals. Phys. Rev, 97(3):660-665, 1985.

[75] P. Lowdin. Advances in Quantum Chemistry. Academic Press Inc., New York, 1964.

[76] S. J. Miyake. The Ground state of the optical polaron in the strong-coupling case. J. Phys. Soc. Jpn, 41(3):747, 1976.

[77] D. Emin and T. Holstein. Adiabatic Theory of an Electron in a Deformable Continuum. Phys. Rev. Lett., 36(6):323-326, feb 1976.

[78] W. P. Su, J. R. Schrieffer, and A. J. Heeger. Solitons in Polyacetylene. Phys. Rev. Lett., 42(25):1698-1701, jun 1979 .

[79] R. W. Munn and R. Silbey. Theory of electronic transport in molecular crystals. II. Zeroth order states incorporating nonlocal linear electronâĂŞphonon coupling. J. Chem. Phys., 83(4):1843, 1985.

[8o] Y. Zhao, D. W. Brown, and K. Lindenberg. On the Munn-Silbey approach to nonlocal exciton-phonon coupling. J. Chem. Phys., 100(3):2335, 1994.

[81] R. G. Della Valle, E. Venuti, L. Farina, A. Brillante, M. Masino, and A. Girlando. Intramolecular and Low-Frequency Intermolecular Vibrations of Pentacene Polymorphs as a Function of Temperature. J. Phys. Chem. B, 108(6):1822-1826, feb 2004.

[82] M. Kuwabara, Y. Ono, and A. Terai. Motion of Charged Soliton in Polyacetylene Due to Electric Field. II. Behavior of Width. J. Phys. Soc. Japan, 6o(4):1286-1293, apr 1991.

[83] Y. Ono and A. Terai. Motion of Charged Soliton in Polyacetylene Due to Electric Field. J. Phys. Soc. Japan, 59(8):2893-2904, aug 1990. 
[84] P. Ma and S. L. Dudarev. Longitudinal magnetic fluctuations in Langevin spin dynamics. Phys. Rev. B, 86(5):054416, 2012.

[85] F. Körmann, A. Breidi, S. L. Dudarev, N. Dupin, G. Ghosh, T. Hickel, P. Korzhavyi, J. A. Muñoz, and I. Ohnuma. Lambda transitions in materials science: Recent advances in CALPHAD and first-principles modelling. Phys. Status Solidi B, 251(1):53-80, 2014.

[86] M. Uhl and J. Kübler. Exchange-Coupled Spin-Fluctuation Theory: Application to Fe, Co, and Ni. Phys. Rev. Lett., 77(2):334-337, jul 1996.

[87] N. M. Rosengaard and B. Johansson. Finite-temperature study of itinerant ferromagnetism in Fe, Co, and Ni. Phys. Rev. B, 55(22):14975-14986, 1997.

[88] A. V. Ruban, S. Khmelevskyi, P. Mohn, and B. Johansson. Temperature-induced longitudinal spin fluctuations in Fe and Ni. Phys. Rev. B - Condens. Matter Mater. Phys., 75(5):1-7, 2007.

[89] A. L. Wysocki, J. K. Glasbrenner, and K. D. Belashchenko. Thermodynamics of itinerant magnets in a classical spin-fluctuation model. Phys. Rev. B, 78(18):1-7, 2008.

[9o] M. Y. Lavrentiev, D. Nguyen-Manh, and S. L. Dudarev. Magnetic cluster expansion model for bcc-fcc transitions in Fe and Fe-Cr alloys. Phys. Rev. B, 81(18):1-6, 2010.

[91] J. B. Staunton and B. L. Gyorffy. Onsager cavity fields in itinerant-electron paramagnets. Phys. Rev. Lett., 69(2):371-374, 1992.

[92] I.A. Abrikosov, A.V. Ponomareva, P. Steneteg, S.A. Barannikova, and B. Alling. Recent progress in simulations of the paramagnetic state of magnetic materials. Curr. Opin. Solid State Mater. Sci., 20(2):85-106, apr 2016.

[93] J. Hellsvik, B. Skubic, L. Nordström, B. Sanyal, O. Eriksson, P. Nordblad, and P. Svedlindh. Dynamics of diluted magnetic semiconductors from atomistic spin-dynamics simulations: Mn-doped GaAs. Phys. Rev. B, 78(14):1-10, 2008.

[94] L. H. Thomas. The calculation of atomic fields. Math. Proc. Cambridge Philos. Soc., 23(05):542, 1927.

[95] E. Fermi. Un metodo statistico per la determinazione di alcune priorieta dell'atome. Accad. Naz. Lincei, 71:1253-1266, 1927.

[96] P. Hohenberg and W. Kohn. Inhomogeneous Electron Gas. Phys. Rev., 136(3B):B864-B871, nov 1964.

[97] W. Kohn and L. J. Sham. Self-Consistent Equations Including Exchange and Correlation Effects. Phys. Rev., 140(4A):A1133-A1138, nov 1965.

[98] D. M. Ceperley and B. J. Alder. Ground State of the Electron Gas by a Stochastic Method. Phys. Rev. Lett., 45(7):566-569, aug 1980.

[99] S. H. Vosko, L. Wilk, and M. Nusair. Accurate spin-dependent electron liquid correlation energies for local spin density calculations: a critical analysis. Can. J. Phys., 58(8):1200-1211, 1980.

[10o] J. P. Perdew and A. Zunger. Self-interaction correction to density-functional approximations for many-electron systems. Phys. Rev. B, 23(10):5048-5079, 1981.

[101] U. von Barth and L. Hedin. A local exchange-correlation potential for the spin polarized case: I. J. Phys. C Solid State Phys., 5(13):1629-1642, 1972.

[102] J. P. Perdew, K. Burke, and M. Ernzerhof. Generalized Gradient Approximation Made Simple. Phys. Rev. Lett., 77(18):3865-3868, 1996.

[103] V. I. Anisimov, J. Zaanen, and O. K. Andersen. Band theory and Mott insulators: Hubbard U instead of Stoner I. Phys. Rev. B, 44(3):943-954, jul 1991.

[104] V. I. Anisimov, I. V. Solovyev, M. A. Korotin, M. T. Czyżyk, and G. A. Sawatzky. Density-functional theory and NiO photoemission spectra. Phys. Rev. B, 48(23):16929-16934, dec 1993. 
80 theoretical description of the electron-lattice interaction in molecular and magnetic crystals

[105] V. I. Anisimov, F. Aryasetiawan, and A. I. Liechtenstein. First-principles calculations of the electronic structure and spectra of strongly correlated systems: the LDA + U method. J. Phys. Condens. Matter, 9(4):767-808, 1997.

[106] J. Hubbard. Electron Correlations in Narrow Energy Bands. III. An Improved Solution. Proc. R. Soc. A Math. Phys. Eng. Sci., 281(1386):401-419, sep 1964.

[107] S. L. Dudarev, G. A. Botton, S. Y. Savrasov, C. J. Humphreys, and A. P. Sutton. Electron-energy-loss spectra and the structural stability of nickel oxide: An LSDA+U study. Phys. Rev. B, 57(3):1505-1509, jan 1998.

[108] D. Marx and J. Hutter. Ab initio molecular dynamics: Theory and implementation. Mod. methods algorithms quantum Chem., 1:301-449, 2000.

[109] B. L. Gyorffy and G. M. Stocks. Concentration waves and Fermi surfaces in random metallic alloys. Phys. Rev. Lett., 50(5):374-377, 1983.

[110] J. B. Staunton, D. D. Johnson, and F. J. Pinski. Compositional short-range ordering in metallic alloys: Band-filling, charge-transfer, and size effects from a first-principles all-electron Landau-type theory. Phys. Rev. B, 50(3):1450-1472, jul 1994.

[111] J. Korringa. On the calculation of the energy of a Bloch wave in a metal. Physica, 13(6-7):392-400, 1947.

[112] W. Kohn and N. Rostoker. Solution of the schrödinger equation in periodic lattices with an application to metallic lithium. Phys. Rev., 94(5):1111-1120, 1954.

[113] A. M. N. Niklasson, J. M. Wills, M. I. Katsnelson, I. A. Abrikosov, O. Eriksson, and B. Johansson. Modeling the actinides with disordered local moments. Phys. Rev. B, 67(23):235105, 2003.

[114] J. Hubbard. Electron Correlations in Narrow Energy Bands. III. An Improved Solution. Proc. R. Soc. A Math. Phys. Eng. Sci., 281(1386):401-419, sep 1964.

[115] B. Alling, T. Marten, and I. A. Abrikosov. Effect of magnetic disorder and strong electron correlations on the thermodynamics of CrN. Phys. Rev. B, 82(18):184430, nov 2010.

[116] A. Zunger, S. H. Wei, L. G. Ferreira, and J. E. Bernard. Special quasirandom structures. Phys. Rev. Lett., 65(3):353-356, jul 1990.

[117] A. Filippetti and N. A. Hill. Magnetic Stress as a Driving Force of Structural Distortions: The Case of CrN. Phys. Rev. Lett., 85(24):5166-5169, dec 2000.

[118] A. V. Ruban and I. A. Abrikosov. Configurational thermodynamics of alloys from first principles: effective cluster interactions. Reports Prog. Phys., 71(4):046501, 2008.

[119] A. V. Ruban and V. I. Razumovskiy. Spin-wave method for the total energy of paramagnetic state. Phys. Rev. B, 85(17):174407, may 2012.

[120] O. Hellman, I. A. Abrikosov, and S. I. Simak. Lattice dynamics of anharmonic solids from first principles. Phys. Rev. B, 84(18):180301, nov 2011.

[121] O. Hellman. Thermal properties of materials from first principles. PhD thesis, 2012.

[122] O. Hellman, P. Steneteg, I. A. Abrikosov, and S. I. Simak. Temperature dependent effective potential method for accurate free energy calculations of solids. Phys. Rev. B, 87(10):104111, mar 2013.

[123] N. Shulumba, O. Hellman, L. Rogström, Z. Raza, F. Tasnádi, I. A. Abrikosov, and M. Odén. Temperature-dependent elastic properties of Ti1-xAlxN alloys. Appl. Phys. Lett., 107(23):231901, dec 2015 .

[124] N. Shulumba. Vibrations in solids: From first principles lattice dynamics to high temperature phase stability Typeset using L. PhD thesis, 2015. 
[125] G. Kresse and J. Furthmüller. Efficiency of ab-initio total energy calculations for metals and semiconductors using a plane-wave basis set. Comput. Mater. Sci., 6(1):15-50, jul 1996.

[126] G. Kresse and J. Furthmüller. Efficient iterative schemes for ab initio total-energy calculations using a plane-wave basis set. Phys. Rev. B, 54(16):11169-11186, oct 1996.

[127] G. Kresse and J. Hafner. Ab initio molecular dynamics for open-shell transition metals. Phys. Rev. B, 48(17):13115-13118, nov 1993.

[128] G. Kresse and D. Joubert. From ultrasoft pseudopotentials to the projector augmented-wave method. Phys. Rev. B, 59(3):1758-1775, jan 1999.

[129] M. Riedmiller and H. Braun. A direct adaptive method for faster backpropagation learning: the RPROP algorithm. In IEEE Int. Conf. Neural Networks, pages 586-591. IEEE, 1993.

[130] S. Stafström. Electron localization and the transition from adiabatic to nonadiabatic charge transport in organic conductors. Chem. Soc. Rev., 39(7):2484-99, jul 2010.

[131] D. Emin. Phonon-assisted transition rates I. Optical-phonon-assisted hopping in solids. Adv. Phys., 24(3):305, 1975 .

[132] E. Mozafari and S. Stafström. Polaron stability in molecular crystals. Phys. Lett. A, 376(22):1807-1811, apr 2012.

[133] A. Ambainis and O. Regev. An Elementary Proof of the Quantum Adiabatic Theorem. arXiv, page 12, 2004 .

[134] Anderson, P. W. Absence of Diffusion in Certain Random Lattices. Phys. Rev., 109(5):1492-1505, mar 1958.

[135] H. J. C. Berendsen, J. P. M. Postma, W. F. van Gunsteren, A. DiNola, and J. R. Haak. Molecular dynamics with coupling to an external bath. J. Chem. Phys., 81(8):3684, 1984.

[136] L. Wen, L. Yuan, Q. Zhen, G. Kun, Y. Sun, and L. De-Sheng. Effect of Temperature on Polaron Stability in a One-Dimensional Organic Lattice. Chin. Phys. Lett., 26(3):037101, mar 2009.

[137] L. A. Ribeiro, P. Neto, W. da Cunha, L. F. Roncaratti, R. Gargano, F. da Silva, and G. M. e Silva. Exciton dissociation and charge carrier recombination processes in organic semiconductors. J. Chem. Phys., 135(22):224901, dec 2011.

[138] A. Brunger, C. L. Brooks, and M. Karplus. Stochastic boundary conditions for molecular dynamics simulations of ST2 water. Chem. phys. lett., 105(5):495-500, mar 1984.

[139] J. A. Izaguirre, D. P. Catarello, J. M. Wozniak, and R. D. Skeel. Langevin stabilization of molecular dynamics. J. Chem. Phys., 114(5):2090, 2001.

[140] G. Kalosakas, S. Aubry, and G. Tsironis. Polaron solutions and normal-mode analysis in the semiclassical Holstein model. Phys. Rev. B, 58(6):3094-3104, aug 1998.

[141] E. Mozafari, B. Alling, P. Steneteg, and Igor A. Abrikosov. Role of N defects in paramagnetic $\mathrm{CrN}$ at finite temperatures from first principles. Phys. Rev. B, 91(9):094101, mar 2015.

[142] N. Shulumba, B. Alling, O. Hellman, E. Mozafari, P. Steneteg, M. Odén, and I. A. Abrikosov. Vibrational free energy and phase stability of paramagnetic and antiferromagnetic $\mathrm{CrN}$ from ab initio molecular dynamics. Phys. Rev. B, 89(17):174108, may 2014.

[143] S. Wang, X. Yu, J. Zhang, M. Chen, J. Zhu, L. Wang, D. He, Z. Lin, R. Zhang, K. Leinenweber, and Y. Zhao. Experimental invalidation of phase-transition-induced elastic softening in CrN. Phys. Rev. B, 86(6):064111, aug 2012.

[144] P. Steneteg, O. Hellman, O. Vekilova, N. Shulumba, F. Tasnádi, and I. A. Abrikosov. Temperature dependence of TiN elastic constants from ab initio molecular dynamics simulations. Phys. Rev. B, 87(9):094114, mar 2013. 
[145] O. Vekilova. Influence of stresses and impurities on thermodynamic and elastic properties of metals and alloys from ab initio theory. PhD thesis, Linköping University, 2013.

[146] H. Holleck. Material selection for hard coatings. J. Vac. Sci. Technol. A Vacuum, Surfaces, Film., 4(6):2661, nov 1986.

[147] B.H. Brandow. Electronic structure of Mott insulators. Adv. Phys., 26(5):651-808, sep 1977.

[148] G. A. Sawatzky and J. W. Allen. Magnitude and Origin of the Band Gap in NiO. Phys. Rev. Lett., 53(24):2339-2342, dec 1984 .

[149] K. Terakura, A. R. Williams, T. Oguchi, and J. Kübler. Transition-Metal Monoxides: Band or Mott Insulators. Phys. Rev. Lett., 52(20):1830-1833, may 1984.

[150] K. Terakura, T. Oguchi, A. R. Williams, and J. Kübler. Band theory of insulating transition-metal monoxides: Band-structure calculations. Phys. Rev. B, 3o(8):4734-4747, oct 1984.

[151] S. Hufner, P. Steiner, I. Sander, F. Reinert, and H. Schmitt. The optical gap on NiO. Z. Phys. B Condens. Matter, 86:207-215, 1992.

[152] R. Zimmermann, P. Steiner, R. Claessen, F. Reinert, S. Hüfner, P. Blaha, and P. Dufek. Electronic structure of $3 \mathrm{~d}$-transition-metal oxides: on-site Coulomb repulsion versus covalency. J. Phys. Condens. Matter, 11(7):1657-1682, feb 1999.

[153] X. Ren, I. Leonov, G. Keller, M. Kollar, I. Nekrasov, and D. Vollhardt. LDA+DMFT computation of the electronic spectrum of NiO. Phys. Rev. B, 74(19):195114, nov 2006.

[154] E. Z. Kurmaev, R. G. Wilks, A. Moewes, L. D. Finkelstein, S. N. Shamin, and J. Kuneš. Oxygen x-ray emission and absorption spectra as a probe of the electronic structure of strongly correlated oxides. Phys. Rev. B, 77(16):165127, apr 2008.

[155] R. Gillen and J. Robertson. Accurate screened exchange band structures for the transition metal monoxides $\mathrm{MnO}, \mathrm{FeO}, \mathrm{CoO}$ and $\mathrm{NiO}$. J. Phys. Condens. Matter, 25(16):165502, apr 2013.

[156] F. Manghi, C. Calandra, and Stefano Ossicini. Quasiparticle Band Structure of NiO: The MottHubbard Picture Regained. Phys. Rev. Lett., 73(23):3129-3132, dec 1994. 


\section{Included Publications and Author's Contribution}

\section{Paper I}

Title

Polaron stability in molecular crystals

Authors

E. Mozafari, S. Stafström

\section{Publication}

Physics Letters A 376(22):1807, 2012.

\section{Contribution}

The code is developed by me. I performed all the calculations and jointly wrote the article.

\section{Paper II}

Title

Polaron dynamics in a two-dimentional Holstein-Peierls system

Authors

E. Mozafari, S. Stafström

\section{Publication}

The Journal of Chemical Physics 138(18):184104, 2013.

\section{Contribution}

I developed the code and performed all the numerical calculations. The paper is written jointly.

\section{Paper III}

Title

Role of $\mathrm{N}$ defects in paramagnetic $\mathrm{CrN}$ at finite temperatures from first principles 
84 theoretical description of the electron-lattice interaction in molecular and magnetic crystals

\section{Authors}

E. Mozafari, B. Alling, P. Steneteg and I. A. Abrikosov

\section{Publication}

Physical Review B 91:094101, 2015.

\section{Contribution}

I performed all the calculations and analyzed the data. The article is written jointly.

\section{Paper IV}

Title

Finite-temperature elastic constants of paramagnetic materials within the diordered local moments picture from $a b$ initio molecular dynamics calculations

\section{Authors}

E. Mozafari, N. Shulumba, P. Steneteg, B. Alling and I. A. Abrikosov

\section{Publication}

Physical Review B 94:054111, 2016.

\section{Contribution}

I performed all the DLM-MD calculations, analyzed the data and jointly wrote the manuscript.

\section{Paper V}

Title

Vibrational free energy and phase stability of paramagnetic and antiferromagnetic $\mathrm{CrN}$ from ab-initio molecular dynamics

\section{Authors}

N. Shulumba, B. Alling, O. Hellman, E. Mozafari, P. Steneteg, M. Odén and I. A. Abrikosov

\section{Publication}

Physical Review B 89:174108, 2014.

\section{Contribution}

I performed some of the DLM-MD calculations and took part in writing the article. 


\title{
Paper VI
}

\section{Title}

Effect of lattice dynamics on the electronic structure of paramagnetic $\mathrm{NiO}$ within Disordered Local Moment picture

\author{
Authors \\ E. Mozafari, B. Alling, M. P.Belov and I. A. Abrikosov \\ Publication \\ In manuscript. \\ Contribution
}

I performed all the DLM-MD calculations, analyzed the data and jointly wrote the manuscript. 



\section{Papers}

The articles associated with this thesis have been removed for copyright reasons. For more details about these see:

http://urn.kb.se/resolve?urn=urn:nbn:se:liu:diva-130517 